\title{
Summary of the CMS potential for the Higgs boson discovery
}

\section{Journal Article}

\section{Author(s):}

Abdullin, Salavat; Dittmar, Michael; et al.

Publication date:

2005-02

Permanent link:

https://doi.org/10.3929/ethz-b-000034674

Rights / license:

Creative Commons Attribution 4.0 International

\section{Originally published in:}

The European Physical Journal C 39, https://doi.org/10.1140/epjcd/s2004-02-003-9 


\title{
Summary of the CMS potential for the Higgs boson discovery
}

\author{
S. Abdullin ${ }^{1, a}$, S. Banerjee ${ }^{2}$, L. Bellucci ${ }^{3}$, C. Charlot $^{4}$, D. Denegri ${ }^{5, b}$, M. Dittmar ${ }^{6}$, V. Drollinger ${ }^{7}$, M.N. Dubinin ${ }^{8}$, \\ M. Dzelalija ${ }^{9}$, D. Green ${ }^{10}$, I. Iasvili ${ }^{10}$, V.A. Ilyin ${ }^{8}$, R. Kinnunen ${ }^{11}$, S. Kunori ${ }^{1}$ K. Lassila-Perini ${ }^{11}$, S. Lehti ${ }^{11}$, \\ K. Mazumdar ${ }^{2}$, F. Moortgat ${ }^{12}$, Th. Muller ${ }^{13}$, A. Nikitenko ${ }^{14, a}$, I. Puljak ${ }^{9}$, P. Salmi ${ }^{11}$, C. Seez ${ }^{14}$, S. Slabospitsky ${ }^{15}$, \\ N. Stepanov ${ }^{5, a}$, R. Vidal ${ }^{10}$, W. Wu ${ }^{10}$, H.D. Yildiz ${ }^{16}$, M. Zeyrek ${ }^{16}$ \\ 1 University of Maryland, College Park, Maryland, USA \\ 2 Tata Institute of Fundamental Research, Mumbai, India \\ 3 Universita di Firenze e Sezione dell'INFN, Firenze, Italia \\ 4 Ecole Polytechnique, IN2P3-CRNS, Palaiseau, France \\ 5 European Laboratory for Nuclear Research (CERN) \\ 6 Eidgenössische Technische Hochschule Zurich/ETH, Zurich, Switzerland \\ 7 Physics Department of Padua University, Padova, Italy \\ 8 Institute of Nuclear Physics, Moscow State University, Moscow, Russia \\ 9 University of Split, Split, Croatia \\ 10 Fermi National Accelerator Laboratory, Batavia, Illinois, USA \\ 11 Helsinki Institute of Physics, Helsinki, Finland \\ 12 Department of Physics, University of Antwerpen, Antwerpen; Research Assistant FWO-Flanders, Belgium \\ 13 IEKP, Karlsruhe University, Germany \\ 14 Imperial College, University of London, London, UK \\ 15 IHEP, Protvino, Moscow Region, Russia \\ 16 Middle East Technical University, Ankara, Turkey
}

Received: 9 April 2004 / Revised version: 26 November 2004 / Accepted: 29 November 2004 / Published online: 23 February 2005 - (C) Springer-Verlag / Società Italiana di Fisica 2005

\begin{abstract}
This work summarizes the studies for the Higgs boson searches in CMS at the LHC collider. The main discovery channels are presented and the potential is given for the discovery of the SM Higgs boson and the Higgs bosons of the MSSM. The phenomenology, detector, trigger and reconstruction issues are briefly discussed.
\end{abstract}

\section{Introduction}

The studies for the Higgs bosons searches in CMS are mainly performed in the framework of the Standard Model (SM) and its Minimal Supersymmetric extension (MSSM). In the SM Higgs boson mechanism, the Higgs boson mass $\mathrm{m}_{\mathrm{H}}$ is the only free parameter. The MSSM contains five Higgs bosons: the lighter scalar h, the heavier scalar $\mathrm{H}$, the pseudoscalar $\mathrm{A}$ and the two charged bosons $\mathrm{H}^{ \pm}$. A general agreement is to present the MSSM parameter space as a function of the pseudoscalar mass $\mathrm{m}_{\mathrm{A}}$ and the ratio $\tan \beta$ of the vacuum expectation values of the two Higgs boson doublets. For the SUSY parameters, the following values of the $\mathrm{m}_{\mathrm{h}}^{\max }$ scenario [1] (used in the LEP studies) are taken unless stated otherwise: $\mathrm{M}_{2}=$ $200 \mathrm{GeV} / c^{2}, \mu=-200 \mathrm{GeV} / c^{2}, \mathrm{M}_{\tilde{\mathrm{g}}}=800 \mathrm{GeV} / c^{2}, \mathrm{M}_{\tilde{\mathrm{q}}, \tilde{\ell}}$

\footnotetext{
a On leave from ITEP, Moscow, Russia

b DAPNIA/SPP, CE Saclay, Gif-sur-Yvette, France
}

$=1 \mathrm{TeV} / c^{2}$. The stop mixing parameter is defined as $\mathrm{X}_{\mathrm{t}}$ $=A_{t}-\mu \cot \beta$, with $A_{t}$ being the trilinear Higgs-stop coupling. In the $\mathrm{m}_{\mathrm{h}}^{\max }$ scenario the light Higgs mass reaches its maximal value at $X_{t}=\sqrt{6} \times M_{\text {SUSY }}$, where $M_{S U S Y}$ is the heavy SUSY scale. In this work, the value of $A_{t}$ was fixed to $\sqrt{6} \times \mathrm{M}_{\mathrm{SUSY}}$ with $\mathrm{M}_{\mathrm{SUSY}}=1 \mathrm{TeV} / c^{2}$. Therefore, $\mathrm{X}_{\mathrm{t}}$ varies between $2550 \mathrm{GeV} / c^{2}$ and $2454 \mathrm{GeV} / c^{2}$ for $2<\tan \beta<50$. With respect to the $\mathrm{m}_{\mathrm{h}}^{\max }$ scenario, this choice leads to a small deviation in $\mathrm{m}_{\mathrm{h}}$ and in the final event rates for a given $\tan \beta$. The top mass is set to $175 \mathrm{GeV} / c^{2}$.

At tree level the $\mathrm{h}(\mathrm{H})$ mass is bound to be below(above) the $\mathrm{Z}$ boson mass but the radiative corrections, proportional to $\mathrm{m}_{\mathrm{top}}^{4}$, bring the upper (lower) bound to a significantly larger value. The one loop and dominant two loop calculations, with the SUSY parameters listed above, predict $m_{h}=127 \mathrm{GeV} / c^{2}$ for $A_{t}=2450 \mathrm{GeV} / c^{2}$ and $113 \mathrm{GeV} / c^{2}$ for $\mathrm{A}_{\mathrm{t}}=0$ [2]. 
For several years, LEP and the Tevatron have been operating in an energy range allowing the Higgs boson(s) to be directly and indirectly searched for. Although tantalizing hints were observed around $115-116 \mathrm{GeV} / c^{2}$, the LEP programme was terminated without conclusive evidence for the Higgs boson. The measurements yield a lower bounds of $114.4 \mathrm{GeV} / c^{2}$ for the mass of the SM Higgs boson [3] and 91.0 and $91.9 \mathrm{GeV} / c^{2}$ for the masses of the $\mathrm{h}$ and A bosons in the MSSM [4]. The excluded $\tan \beta$ regions are $0.5<\tan \beta<2.4$ for the maximal $\mathrm{m}_{\mathrm{h}}$ scenario and $0.7<$ $\tan \beta<10.5$ for the no-stop-mixing scenario [4]. The fits to all LEP, SLC and Tevatron precision measurements lead to an indirect prediction for the SM Higgs boson mass of $\mathrm{m}_{\mathrm{H}}=96_{-38}^{+60} \mathrm{GeV} / c^{2}$ and to a $95 \% \mathrm{CL}$ upper limit of $219 \mathrm{GeV} / c^{2}[5]$.

This paper summarizes the CMS studies for the SM and MSSM Higgs bosons. In these studies, performed mainly with fast detector simulation, the main detector dependent issues like mass resolutions and efficiencies for reconstruction and $\mathrm{b}$ tagging were studied with full simulation and used directly or parametrized in the fast simulation. Studies based solely on the full simulation and complete reconstruction are presently in progress for all the main channels with the now frozen detector design. In this paper, the production processes, the decay channels, the search strategy and the influence of the MSSM parameters are discussed in Sects. 2.1 to 2.4. The detector is shortly presented in Sect. 3.1 and the trigger and reconstruction issues are discussed in Sects. 3.2 and 3.3. Section 3.4 is devoted to background simulations. The searches for a SM-like scalar Higgs boson are discussed in Sect. 4.1, those for the neutral Higgs bosons H and A in Sect. 4.2 and the charged Higgs bosons $\mathrm{H}^{ \pm}$in Sect. 4.3. Sections 4.4 and 4.5 summarize the discovery potentials in the SM and the MSSM. The specific SUSY searches, the Higgs boson decays to gauginos, the Higgs boson production through gaugino decays and the discovery potential in the specific SUSY searches are discussed in the Sects. 5.1 and 5.2. The conclusions are given in Sect. 6.

\section{Phenomenology}

\subsection{Higgs boson production processes}

Figure 1 shows the production cross sections for the SM Higgs boson as a function of the Higgs boson mass for different production processes calculated at QCD leading (LO) order with the programs of [6]. The production is dominated by the gluon-gluon fusion, gg $\rightarrow \mathrm{H}$, over the mass range $100 \mathrm{GeV} / c^{2} \lesssim \mathrm{m}_{\mathrm{H}} \lesssim 1 \mathrm{TeV} / c^{2}$. The associated production processes, qq $\rightarrow \mathrm{HW}$, qq $\rightarrow \mathrm{HZ}$, $\mathrm{gg} / \mathrm{qq} \rightarrow \mathrm{t} \overline{\mathrm{t}} \mathrm{H}$ and $\mathrm{gg} / \mathrm{qq} \rightarrow \mathrm{b} \overline{\mathrm{b}} \mathrm{H}$ have cross sections lower by a factor of $\sim 20$ at $\mathrm{m}_{\mathrm{H}} \sim 100 \mathrm{GeV} / c^{2}$ and by a factor of $\sim 1000$ at large masses, $\mathrm{m}_{\mathrm{H}} \gtrsim 500 \mathrm{GeV} / c^{2}$. The production cross section for the gauge boson fusion, $\mathrm{qq} \rightarrow \mathrm{qqH}$, is about $10 \%$ of the $\mathrm{LO}$ production cross section for $\mathrm{gg} \rightarrow \mathrm{H}$ for $\mathrm{m}_{\mathrm{H}} \lesssim 200 \mathrm{GeV} / c^{2}$, and becomes comparable for $\mathrm{m}_{\mathrm{H}} \sim 1 \mathrm{TeV} / c^{2}$.

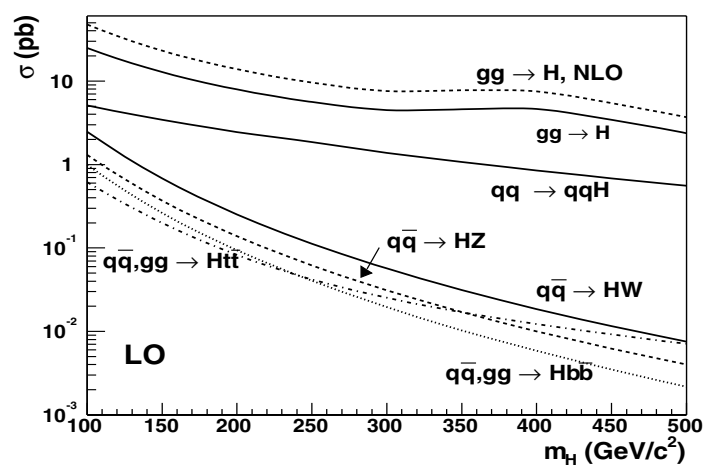

Fig. 1. The leading order (LO) cross sections calculated with the programs of [6] for the SM Higgs boson as a function of $\mathrm{m}_{\mathrm{H}}$. The cross section for the $\mathrm{gg} \rightarrow \mathrm{H}$ process is also shown at NLO

In the MSSM, the lighter scalar $\mathrm{h}$ is SM-like for $\mathrm{m}_{\mathrm{A}}>$ $\mathrm{m}_{\mathrm{h}}^{\max }$ (decoupling region), with production cross sections and decay partial widths close to those of the SM Higgs boson. At large $\tan \beta$, the couplings of the heavy neutral Higgs bosons to the electroweak gauge bosons are strongly suppressed, while those to the down-type fermions are enhanced with $\tan \beta$. The production of the heavy neutral MSSM Higgs bosons $\mathrm{H}$ and A proceeds mainly through $\mathrm{gg} \rightarrow \mathrm{H} / \mathrm{A}$ and $\mathrm{gg} / \mathrm{qq} \rightarrow \mathrm{b} \overline{\mathrm{b}} \mathrm{H} / \mathrm{A}$. At large $\tan \beta$, the $\mathrm{b} \overline{\mathrm{b}} \mathrm{H} / \mathrm{A}$ associated production dominates and is about $90 \%$ of the total rate for $\tan \beta \gtrsim 10$ and $\mathrm{m}_{\mathrm{A}} \gtrsim 300 \mathrm{GeV} / c^{2}$. The heavy scalar Higgs boson is SM-like near its lower mass bound (i.e. at small $\mathrm{m}_{\mathrm{A}}$ and large $\tan \beta$ ) and has in this region a significant cross section in the gauge boson fusion process qq $\rightarrow$ qqH.

If the charged Higgs bosons are light, $\mathrm{m}_{\mathrm{H}^{ \pm}}<\mathrm{m}_{\text {top }}$, they are predominantly produced in $t \bar{t}$ events, with a $t \rightarrow$ $\mathrm{H}^{ \pm} \mathrm{b}$ decay. Several processes, like $\mathrm{gb} \rightarrow \mathrm{tH}^{ \pm}, \mathrm{gg} \rightarrow \mathrm{tbH}^{ \pm}$, $\mathrm{qq}^{\prime} \rightarrow \mathrm{H}^{ \pm}$, gg $\rightarrow \mathrm{H}^{+} \mathrm{H}^{-}$and $\mathrm{gg} \rightarrow \mathrm{W}^{ \pm} \mathrm{H}^{\mp}$ contribute to the production of heavier $\left(\mathrm{m}_{\mathrm{H}^{ \pm}} \geq \mathrm{m}_{\mathrm{t}}\right)$ charged Higgs bosons. The cross section for the production in association with one top quark gb $\rightarrow \mathrm{tH}^{ \pm}$is sizeable $(\sim 0.1 \mathrm{pb}$ for $\left.\mathrm{m}_{\mathrm{H}^{ \pm}}=400 \mathrm{GeV} / c^{2}, \tan \beta=30\right)[7]$. The direct production, $\mathrm{q} \overline{\mathrm{q}} \rightarrow \mathrm{H}^{ \pm}$, has a cross section comparable to that of $\mathrm{gb} \rightarrow$ $\mathrm{tH}^{ \pm}$[8] while for the gg $\rightarrow \mathrm{H}^{+} \mathrm{H}^{-}$and gg $\rightarrow \mathrm{W}^{ \pm} \mathrm{H}^{\mp}$ processes the cross sections are smaller typically by two orders of magnitude [9].

The $\tan ^{2} \beta$ dependence of the cross sections of the associated production processes $\mathrm{gg} \rightarrow \mathrm{b} \overline{\mathrm{b}} \mathrm{H} / \mathrm{A}$ and $\mathrm{gg} \rightarrow \mathrm{btH}^{ \pm}$ can be exploited to measure the value of $\tan \beta$ from the event rates.

The QCD corrections for the $\mathrm{gg} \rightarrow \mathrm{H}$ process are large, with a next-to-leading (NLO) k factor ranging from 1.5 to 1.8 [10]: the production cross section for this process is shown in Fig. 1. The corrections to NNLO have been shown to improve the convergence of the calculation and to increase the $\mathrm{k}$ factor up to $\sim 2$ [11]. The QCD corrections are smaller for the gauge boson fusion ( $\mathrm{k}$ factor $\sim 1.1)$ and for the associated production processes $\mathrm{qq}^{\prime} \rightarrow \mathrm{HW}, \mathrm{q} \overline{\mathrm{q}} \rightarrow \mathrm{HZ}(\mathrm{k}$ factor $\sim 1.3)$ and $\mathrm{gg} / \mathrm{q} \overline{\mathrm{q}} \rightarrow \mathrm{t} \overline{\mathrm{t}} \mathrm{H}(\mathrm{k}$ factor $\sim 1.2)[10]$. For the production of the charged Higgs bosons, the NLO corrections increase the cross section for 


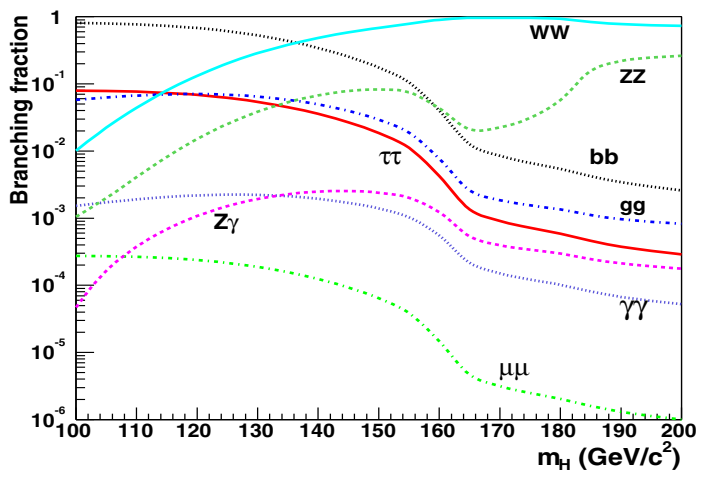

Fig. 2. Branching fractions for the SM Higgs boson as a function of $\mathrm{m}_{\mathrm{H}}$ calculated with HDECAY [12]

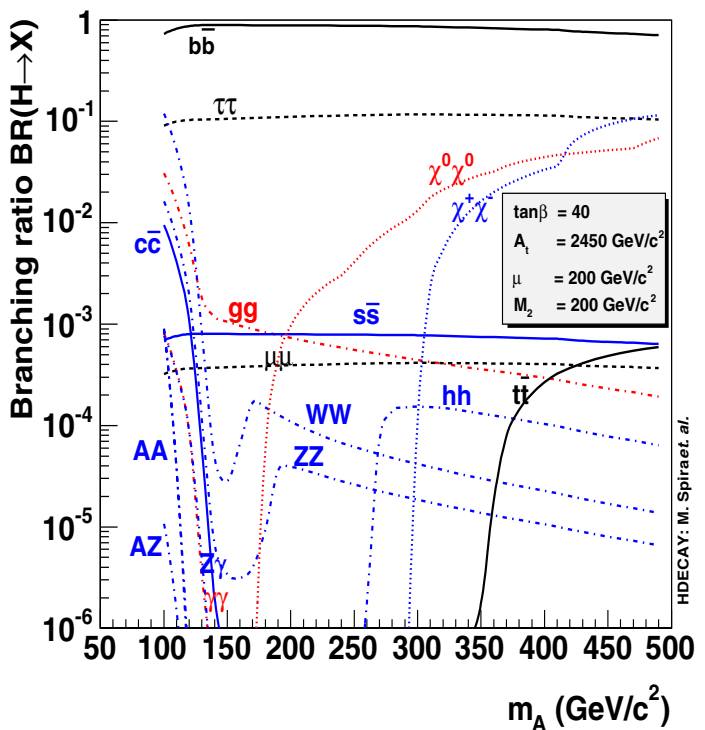

Fig. 3. Branching fractions for the MSSM Higgs boson $\mathrm{H}$ for $\tan \beta=40$ as a function of $\mathrm{m}_{\mathrm{A}}$ with $\mathrm{M}_{2}=200 \mathrm{GeV} / c^{2}, \mu$ $=300 \mathrm{GeV} / c^{2}, \mathrm{M}_{\tilde{\mathrm{g}}}=800 \mathrm{GeV} / c^{2}, \mathrm{M}_{\tilde{\mathrm{q}}, \tilde{\ell}}=1 \mathrm{TeV} / c^{2}$. The branching fractions to gg, $c \bar{c}$ and $s \bar{s}$ are not shown

$\mathrm{gb} \rightarrow \mathrm{tH}^{ \pm}$by about $30 \%$ [7]. In the next sections, the results from NLO analysis are given for the channels where the gluon-gluon fusion dominates the production: $\mathrm{H} \rightarrow \gamma \gamma$, $\mathrm{H} \rightarrow \mathrm{ZZ}^{(*)} \rightarrow \ell^{+} \ell^{-} \ell^{\prime+} \ell^{\prime-}$ and $\mathrm{H} \rightarrow \mathrm{WW}^{(*)} \rightarrow \ell^{+} \ell^{-} \nu \nu$.

\subsection{Higgs boson decay channels}

The branching fractions for the SM-like Higgs boson, calculated with the HDECAY program [12], are shown in Fig. 2 as a function of the Higgs boson mass. For Higgs boson masses below $130 \mathrm{GeV} / c^{2}$, the $\mathrm{H} \rightarrow \mathrm{b} \overline{\mathrm{b}}$ decay channel dominates. In this mass range, the $\mathrm{H} \rightarrow \tau^{+} \tau^{-}$decay rate is also sizeable and amounts to $\sim 8 \%$. The branching fraction for $\mathrm{H} \rightarrow \gamma \gamma$ is about $1.5 \times 10^{-3}$ for $\mathrm{m}_{\mathrm{H}} \lesssim 150 \mathrm{GeV} / c^{2}$, that to $\mathrm{H} \rightarrow \mu^{+} \mu^{-}$about $2 \times 10^{-4}$ for $\mathrm{m}_{\mathrm{H}} \lesssim 130 \mathrm{GeV} / c^{2}$ and the branching fraction to $\mathrm{H} \rightarrow \mathrm{Z} \gamma$ about $2 \times 10^{-3}$ for $140 \mathrm{GeV} / c^{2} \lesssim \mathrm{m}_{\mathrm{H}} \lesssim 150 \mathrm{GeV} / c^{2}$. For larger $\mathrm{m}_{\mathrm{H}}$, the decay is almost entirely through the $\mathrm{H} \rightarrow \mathrm{WW}^{*} / \mathrm{WW}$ and

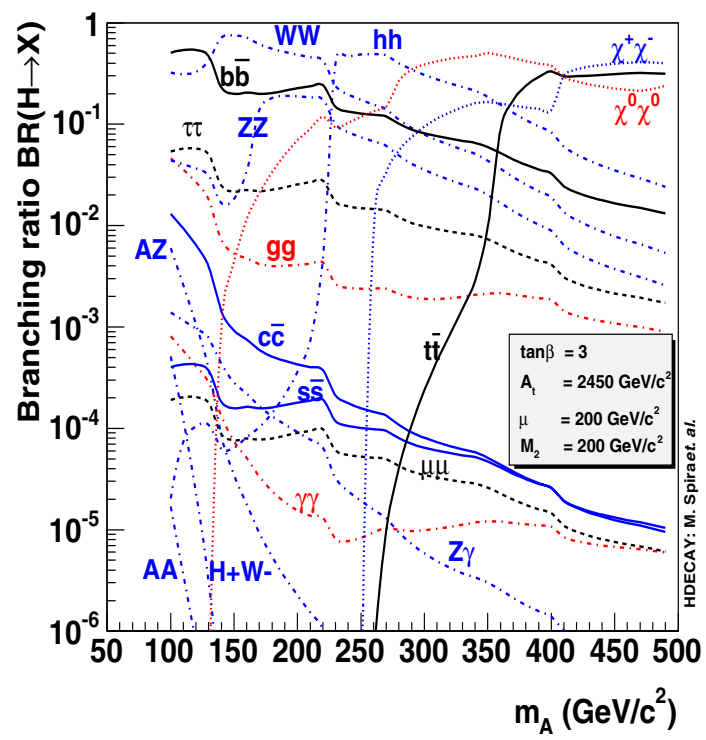

Fig. 4. The same as in Fig. 3 but for $\tan \beta=3$

$\mathrm{H} \rightarrow \mathrm{ZZ} * \mathrm{ZZ}$ channels, although for $\mathrm{m}_{\mathrm{H}} \gtrsim 2 \mathrm{~m}_{\mathrm{top}}$ the $\mathrm{t} \overline{\mathrm{t}}$ branching fraction can be as large as $20 \%$.

Figures 3 and 4 show the branching fractions for the heavy neutral MSSM Higgs boson $\mathrm{H}$ and $\mathrm{A}$ for $\tan \beta=40$ and 3 , respectively. At large $\tan \beta(\gtrsim 10)$ the $\mathrm{H}, \mathrm{A} \rightarrow \mathrm{b} \overline{\mathrm{b}}$ decay dominates, the branching fraction to $\tau^{+} \tau^{-}$is about $10 \%$ and that to $\mu^{+} \mu^{-}$about $3 \times 10^{-4}$. At small $\tan \beta$, the branching fractions for $\mathrm{H} \rightarrow \mathrm{hh}, \mathrm{H} \rightarrow \mathrm{WW}$ and $\mathrm{H} \rightarrow \mathrm{ZZ}$ and $\mathrm{A} \rightarrow \mathrm{hZ}$ can reach $\sim 60 \%$ below the thresholds of the decays to $t \bar{t}$ and to gauginos. The branching fraction to $t \bar{t}$ can be as large as $90 \%$. The branching fractions to $\gamma \gamma$ and $\mathrm{Z} \gamma$ remain below the level of $\sim 10^{-3}$. The decay fractions to gauginos can reach $\sim 20 \%$ at large $\tan \beta$ and $\sim 70 \%$ at small $\tan \beta$ and are sensitive to the SUSY parameters.

The light charged Higgs bosons $\left(\mathrm{m}_{\mathrm{H}^{ \pm}}<\mathrm{m}_{\mathrm{top}}\right)$ decay to $\tau \nu_{\tau}$ with an almost $100 \%$ branching fraction. At large $\tan \beta$, the $\mathrm{H}^{ \pm} \rightarrow$ tb decay dominates for $\mathrm{m}_{\mathrm{H}^{ \pm}} \gtrsim 200 \mathrm{GeV} / c^{2}$, the $\mathrm{H}^{ \pm} \rightarrow \tau \nu_{\tau}$ branching fraction decreases and is about $10 \%$ for $\mathrm{m}_{\mathrm{H}^{ \pm}} \gtrsim 400 \mathrm{GeV} / c^{2}$. The other interesting branching fraction $\mathrm{H}^{ \pm} \rightarrow \mu \nu_{\mu}$ is at the level of only $\sim 10^{-3}$. The branching fraction for the $\mathrm{H}^{ \pm} \rightarrow$ Wh decay mode may reach $\sim 10 \%$ at small $\tan \beta$ around $\mathrm{m}_{\mathrm{H}^{ \pm}} \sim 200 \mathrm{GeV} / c^{2}$. The decay branching fractions to gauginos can reach $\sim 10 \%$ at large $\tan \beta$ and $\sim 30 \%$ at small $\tan \beta$, in the SUSY parameter region where gauginos are predicted to be light enough.

\subsection{Search strategy}

In this section the search strategies are discussed for a light SM-type Higgs boson, a heavy SM Higgs boson, heavy MSSM Higgs bosons and MSSM Higgs bosons in specific SUSY channels, with decays to gauginos and in the production through the gaugino decays. In addition to the Higgs boson mass and decay channel these strategies depend on the production process: the inclusive, the asso- 
ciated production or the production in the weak gauge boson fusion.

\subsubsection{SM Higgs boson}

A light SM-like Higgs boson with $\mathrm{m}_{\mathrm{H}} \lesssim 150 \mathrm{GeV} / \mathrm{c}^{2}$ can be searched for in the $\mathrm{H} \rightarrow \gamma \gamma, \mathrm{H} \rightarrow \mathrm{b} \overline{\mathrm{b}}, \mathrm{H} \rightarrow \mathrm{ZZ}^{*} / \mathrm{WW}^{*}$, $\mathrm{H} \rightarrow \tau^{+} \tau^{-}, \mathrm{H} \rightarrow \mu^{+} \mu^{-}$and $\mathrm{H} \rightarrow \mathrm{Z} \gamma$ decay channels. In this mass range the small natural width, $\Gamma_{\mathrm{H}} \ll 1 \mathrm{GeV}$, can be exploited by optimizing the mass resolutions in the $\mathrm{H} \rightarrow \gamma \gamma, \mathrm{H} \rightarrow \mathrm{ZZ}^{*} \rightarrow \ell^{+} \ell^{-} \ell^{\prime+} \ell^{\prime-}$ and $\mathrm{H} \rightarrow \mu^{+} \mu^{-}$channels. These channels can be searched for inclusively in the dominant $\mathrm{gg} \rightarrow \mathrm{H}$ production process. In this mode, the irreducible background from direct $\gamma \gamma$ production is large for the $\mathrm{H} \rightarrow \gamma \gamma$ channel, while the $\mathrm{H} \rightarrow \mathrm{ZZ}^{*} \rightarrow \ell^{+} \ell^{-} \ell^{\prime+} \ell^{\prime-}$ channel yields a clean signature with a much lower background. The $\mathrm{H} \rightarrow \gamma \gamma$ channel can also be searched for in the associated production processes $\mathrm{WH}$ and $\mathrm{t} \overline{\mathrm{t}} \mathrm{H}$ with an isolated lepton from $\mathrm{W} \rightarrow \ell \nu_{\ell}$ and in the $\mathrm{H}+$ jet production with a large $\mathrm{E}_{\mathrm{T}}$ hadronic jet. Better signal-tobackground ratios are obtained, thereby relaxing the demand on the resolution of the electromagnetic calorimeter, but the event rates are low due to small production cross sections. The large $\mathrm{H} \rightarrow \mathrm{b} \overline{\mathrm{b}}$ branching fraction for $\mathrm{m}_{\mathrm{H}} \lesssim 130 \mathrm{GeV} / c^{2}$ can be exploited only in the associated production channels têtH and $\mathrm{WH}$ due to the large backgrounds from multi-jet events and due to the modest $(\sim 11 \%)$ Higgs boson mass resolution with the $\mathrm{H} \rightarrow \mathrm{b} \overline{\mathrm{b}}$ decay channels. Because of their tiny branching fractions, the $\mathrm{H} \rightarrow \mu^{+} \mu^{-}$and $\mathrm{H} \rightarrow \mathrm{Z} \gamma$ decay channels may be exploited only with integrated luminosities exceeding $100 \mathrm{fb}^{-1}$. The gauge boson fusion production $\mathrm{qq} \rightarrow \mathrm{qqH}$ is shown to be accessible in the mass range $\mathrm{m}_{\mathrm{H}} \lesssim 150 \mathrm{GeV} / c^{2}$ in the $\mathrm{H} \rightarrow \gamma \gamma, \mathrm{H} \rightarrow \mathrm{WW}^{*}, \mathrm{H} \rightarrow \tau^{+} \tau^{-}$and possibly in the $\mathrm{H} \rightarrow \mathrm{b} \overline{\mathrm{b}}$ and $\mathrm{H} \rightarrow \mu^{+} \mu^{-}$decay modes [13]. Because no colour is exchanged in the central hard process, the gauge boson fusion leads to a low jet activity in the central rapidity region. Detecting the forward jets and imposing a veto on jets in the rapidity range between the forward jets largely reduces the $\mathrm{QCD}$ and $\mathrm{t} \overline{\mathrm{t}}$ backgrounds.

In the mass range from 120 to $200 \mathrm{GeV} / c^{2}$, the $\mathrm{H} \rightarrow$ $\mathrm{WW}^{*} / \mathrm{WW} \rightarrow \ell^{+} \nu_{\ell} \ell^{-} \nu_{\ell}$ channel can also be exploited. It requires a good understanding of $t \bar{t}$ and Wtb backgrounds. The event rate is large but only the transverse Higgs boson mass can be reconstructed in this channel due to two neutrinos in the final state. In the mass range above $200 \mathrm{GeV} / c^{2}$, the $\mathrm{H} \rightarrow \mathrm{ZZ} \rightarrow \ell^{+} \ell^{-} \ell^{\prime+} \ell^{\prime-}$ channel yields the highest sensitivity up to $500 \mathrm{GeV} / c^{2}$. Above $500 \mathrm{GeV} / c^{2}$, where the natural width of the Higgs boson is large, the final states with larger branching fractions including jets and $\mathrm{E}_{\mathrm{T}}^{\text {miss }}$ from the $\mathrm{H} \rightarrow \mathrm{ZZ} / \mathrm{WW}$ decays are used. In this mass range, the cross section for the qq $\rightarrow$ qqH production process is large and can be fully exploited.

\subsubsection{Heavy neutral MSSM Higgs bosons $\mathrm{H}$ and $\mathrm{A}$}

The suppression of the couplings to gauge bosons implies different search strategies. At large $\tan \beta$, the coupling enhancement to down-type fermions allows searching for the
$\mathrm{H}$ and $\mathrm{A}$ bosons in the $\mathrm{H}, \mathrm{A} \rightarrow \mu^{+} \mu^{-}, \mathrm{H}, \mathrm{A} \rightarrow \tau^{+} \tau^{-}$and $\mathrm{H}, \mathrm{A} \rightarrow \mathrm{b} \overline{\mathrm{b}}$ decay channels in the associated production $\mathrm{gg} \rightarrow \mathrm{b} \overline{\mathrm{b}} \mathrm{H} / \mathrm{A}$. In this production process, the associated $\mathrm{b}$ jets can be used to suppress the Z, $\gamma^{*}$ and QCD multi-jet backgrounds with b-tagging methods. To take advantage of the hadronic $\tau$ decays in the lepton-plus- $\tau$-jet and two$\tau$-jet final states from the $\mathrm{H}, \mathrm{A} \rightarrow \tau^{+} \tau^{-}$decay, an efficient $\tau$-jet identification method is required to suppress further QCD multi-jet and $\mathrm{W}+$ jet backgrounds. In the next sections, a $\tau$ jet stands for the one or three collimated charged hadrons possibly accompanied by $\pi^{o}$ mesons from a $\tau \rightarrow$ hadrons $+\nu_{\tau}$ decay. The Higgs boson mass can be reconstructed also in the $\mathrm{H}, \mathrm{A} \rightarrow \tau^{+} \tau^{-}$channels from $\mathrm{E}_{\mathrm{T}}^{\text {miss }}$ and the visible $\tau$ momenta exploiting the neutrino collinearity with the parent $\tau$ direction. For the $\mathrm{H}, \mathrm{A} \rightarrow \mathrm{b} \overline{\mathrm{b}}$ decay channel the signal-to-background ratios are low due to overwhelming QCD multi-jet backgrounds. Good understanding of these backgrounds, and b tagging are needed already at the high level trigger.

\subsubsection{Charged MSSM Higgs bosons}

To search for the heavy charged Higgs bosons $\left(\mathrm{m}_{\mathrm{H}^{ \pm}}>\right.$ $\left.\mathrm{m}_{\text {top }}\right)$, the $\mathrm{H}^{ \pm} \rightarrow \tau \nu_{\tau}, \mathrm{H}^{ \pm} \rightarrow$ tb and $\mathrm{H}^{ \pm} \rightarrow$ Wh decay channels can be used in the associated production process gg $\rightarrow \mathrm{btH}^{ \pm}$. The $\mathrm{H}^{ \pm} \rightarrow \tau \nu_{\tau}$ decay channel can be used also in the direct production $\mathrm{q} \overline{\mathrm{q}} \rightarrow \mathrm{H}^{ \pm}$. In the associated production process, the $\mathrm{W}+$ jet and QCD multi-jet backgrounds can be suppressed with $\mathrm{b}$ tagging and reconstruction of the associated top quark. The $\mathrm{H}^{ \pm} \rightarrow \tau \nu_{\tau}$ decay channel is particularly interesting, when hadronic $\tau$

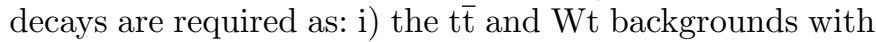
genuine $\tau$ 's can be suppressed exploiting the different helicity correlations in the $\mathrm{H}^{ \pm} \rightarrow \tau \nu_{\tau}$ and the $\mathrm{W}^{ \pm} \rightarrow \tau \nu_{\tau}$ decay channels [14], ii) the transverse Higgs boson mass can be reconstructed allowing the signal and background to be separated. For the $\mathrm{H}^{ \pm} \rightarrow$ tb decay channel, the $t \bar{t}$-plus-multijet backgrounds can be reduced only with $b$ tagging, leading to low signal-to-background ratios. The $\mathrm{H}^{ \pm} \rightarrow \mu \nu_{\mu}$ decay channel may be exploited with integrated luminosities exceeding $100 \mathrm{fb}^{-1}$. The light charged Higgs bosons $\left(\mathrm{m}_{\mathrm{H}^{ \pm}}<\mathrm{m}_{\mathrm{top}}\right)$ can be searched for in the $\mathrm{H}^{ \pm} \rightarrow \tau \nu_{\tau}$ decay channel in the $t \overline{\mathrm{t}}$ production, suppressing the backgrounds with an isolated lepton from the accompanying W decay. For efficient triggering of the $\mathrm{H}^{ \pm} \rightarrow \tau \nu_{\tau}$ channel with a single $\tau$ jet and of the two- $\tau$-jet final state from $\mathrm{H}, \mathrm{A} \rightarrow \tau^{+} \tau^{-}$, a specific trigger algorithm is needed.

\subsubsection{Decays off and into gauginos}

The decays into SM particles may provide access to the heavy MSSM Higgs bosons only at large $\tan \beta$. The small and medium $\tan \beta$ values could be partly explored with Higgs boson decays to gauginos. If the gaugino masses are small enough, the branching fractions for the $\mathrm{H}, \mathrm{A} \rightarrow \chi_{2} \chi_{2}$ and $\mathrm{H}^{ \pm} \rightarrow \chi_{2,3} \chi_{1,2}^{ \pm}$decays can be sizeable. These decay channels can be searched for in multi-lepton final states 
if the sleptons are light enough because, for light sleptons, the branching fraction for the $\chi_{2} \rightarrow \tilde{\ell} \ell \rightarrow \chi_{1} \ell^{+} \ell^{-}$ decay can be as large as $\sim 55 \%$. The Higgs boson mass reconstruction is not possible for these channels due to the invisible neutralinos at the end of the decay chain.

In the MSSM, Higgs bosons can also be produced as decay products of gauginos in the squark and gluino cascades. More specifically, squarks and gluinos can decay to heavy charginos and neutralinos, $\chi_{2}^{ \pm}, \chi_{3}$ and $\chi_{4}$. If kinematically allowed these particles decay into the lighter chargino and neutralinos, $\chi_{1}^{ \pm}, \chi_{1}$ and $\chi_{2}$ and Higgs bosons. Squarks and gluinos can also decay directly to the light gauginos $\chi_{1}^{ \pm}$and $\chi_{2}$ which then decay to the lightest neutralino and Higgs bosons. The production rates can be large $\left(\sim 0.3 \mathrm{pb}\right.$ for $\left.\mathrm{m}_{\mathrm{A}} \sim 200 \mathrm{GeV} / c^{2}\right)$ as the squarks and gluinos are produced via strong interactions. These events are characterized by large $\mathrm{E}_{\mathrm{T}}^{\mathrm{miss}}$ and large jet multiplicity. These features can be used to suppress efficiently the SM backgrounds and allow the $\mathrm{h}, \mathrm{H}, \mathrm{A} \rightarrow \mathrm{b} \overline{\mathrm{b}}$ decays to be exploited.

\subsection{Influence of MSSM parameters}

\subsubsection{Decays to SUSY particles}

The lightest charginos and neutralinos may have masses of the order of the $\mathrm{Z}$ boson mass. The heavy CP-even, CPodd and charged Higgs bosons of the MSSM can therefore decay into these particles. In addition to $\tan \beta$ and $\mathrm{m}_{\mathrm{A}}$, the masses of charginos and neutralinos as well as their couplings to the Higgs bosons depend on the higgsino mass parameter $\mu$ and the gaugino mass parameter $\mathrm{M}_{2}$.

The MSSM Higgs bosons can decay also into the SUSY partners of leptons and quarks if the latter are light enough. In particular, if kinematically allowed, decays into third generation sfermions can be dominant due to enhanced couplings. The sfermions masses and couplings to Higgs bosons for each generation depend on the left- and right-handed soft SUSY-breaking mass parameters $m_{\tilde{f}_{L}}$ and $\mathrm{m}_{\tilde{\mathrm{f}}_{\mathrm{R}}}$ and the trilinear coupling $\mathrm{A}_{\mathrm{t}}$ (in addition to $\tan \beta$ and $\mathrm{m}_{\mathrm{A}}$ ).

The variation of the $\mathrm{H} \rightarrow \tau^{+} \tau^{-}$branching fraction as a function of the MSSM parameters was studied in [15]. For for smaller $|\mu|$ and $\mathrm{M}_{2}$ the neutralinos become lighter, which increases the branching fractions to these particles for large Higgs boson masses and suppresses the $\mathrm{H} \rightarrow \tau^{+} \tau^{-}$branching fraction. For $\mathrm{m}_{\mathrm{A}}=500 \mathrm{GeV} / c^{2}$ and $\tan \beta=30$ the $\mathrm{H} \rightarrow \tau^{+} \tau^{-}$branching fraction decreases by about $20 \%$ when $\mu$ is decreased from $300 \mathrm{GeV} / c^{2}$ to 200 $\mathrm{GeV} / c^{2}$, with the other SUSY parameters set to $\mathrm{M}_{2}=$ $200 \mathrm{GeV} / c^{2}, \mathrm{M}_{\tilde{\mathrm{g}}}=800 \mathrm{GeV} / c^{2}, \mathrm{M}_{\tilde{\mathrm{q}}, \tilde{\ell}}=1 \mathrm{TeV} / c^{2}$ and $\mathrm{A}_{\mathrm{t}}=2450 \mathrm{GeV} / c^{2}$. Increasing $\mu$ from $300 \mathrm{GeV} / c^{2}$ to $500 \mathrm{GeV} / c^{2}$ enhances this branching fraction by about $15 \%$. These variations are somewhat smaller for negative $\mu$ values while they are much larger at smaller $\tan \beta$ values. The variation as a function of $\mathrm{M}_{2}$ is about $10 \%$ for $120<$ $\mathrm{M}_{2}<300 \mathrm{GeV} / c^{2}$. For lighter squarks and gluinos $\left(\mathrm{M}_{\tilde{\mathrm{g}}}=\right.$ $\mathrm{M}_{\tilde{\mathrm{q}}}=500 \mathrm{GeV} / c^{2}$ ), the branching fraction is enhanced by $\sim 14 \%$. At large $\tan \beta$ the $\mathrm{H}, \mathrm{A} \rightarrow \tau^{+} \tau^{-}$and $\mathrm{H}, \mathrm{A} \rightarrow \mu^{+} \mu^{-}$ branching fractions can be affected by stop mixing only if the MSSM mass spectrum is such that the $\mathrm{H}, \mathrm{A} \rightarrow \tilde{\mathrm{t}} \tilde{\mathrm{t}}$ decays are allowed.

\subsubsection{Production of SUSY particles}

The gluon fusion process, gg $\rightarrow \mathrm{h}$, is mediated by loops involving top and bottom quarks as well as squarks. The $\mathrm{h} \rightarrow \gamma \gamma$ decay is mediated by $\mathrm{W}$ and heavy fermion loops as in the Standard Model and, in addition, by charged Higgs, sfermion and chargino loops. If the stop mixing is large, one of the stop quarks can be light. When the stop mass approaches the top mass the destructive interference between the top and stop contributions in the loops becomes significant. As a consequence, the gg $\rightarrow \mathrm{h}$ cross section can be very much reduced while the $h \rightarrow \gamma \gamma$ branching fraction is only slightly enhanced. The net result is a decrease of the overall $\mathrm{gg} \rightarrow \mathrm{h} \rightarrow \gamma \gamma$ rate [16]. This effect can even preclude the observability in the inclusive $\mathrm{h} \rightarrow \gamma \gamma$ and $\mathrm{h} \rightarrow \mathrm{ZZ}^{*} \rightarrow \ell^{+} \ell^{-} \ell^{\prime+} \ell^{\prime-}$ channels for $\mathrm{m}_{\tilde{\mathrm{t}}_{1}} \lesssim 200 \mathrm{GeV} / c^{2}$, and reduce the sensitivity in these channels for $\mathrm{m}_{\tilde{\mathrm{t}}_{1}} \lesssim 300 \mathrm{GeV} / \mathrm{c}^{2}[17]$. The discovery potential for the heavy MSSM Higgs bosons is not sensitive to the stop mixing and light stop $\left(\tilde{\mathrm{t}}_{1}\right)$ mass due to the dominance of associated production (tree diagram) at large $\tan \beta$ and because the CP-odd Higgs boson does not couple to $\tilde{\mathrm{t}}_{1} \tilde{\mathrm{t}}_{1}$ pair but to $\tilde{\mathrm{t}}_{1} \tilde{\mathrm{t}}_{2}\left(\mathrm{~m}_{\tilde{\mathrm{t}}_{2}}\right.$ remains always large, of the order of $\left.1 \mathrm{TeV} / c^{2}\right)$.

\section{Simulation and reconstruction}

\subsection{Detector}

A detailed description of the CMS detector can be found in [17-22]. The distinctive features of the detector are a $4 \mathrm{~T}$ axial magnetic field, a multilayer muon system in the return yoke [18], a scintillating crystal electromagnetic calorimeter (ECAL), a sampling hadron calorimeter (HCAL) and an all-silicon inner tracking system based on fine-grained micro-strip and pixel detectors. The large fraction of the calorimeters and the tracker are located inside the solenoid.

In the original design, the tracking detector [19] consisted of a pixel vertex, a silicon micro-strip detector and MSGC gas detectors in the outer layers. In 2000 a modification of the original design of the tracker was approved [20]. In the new design, the outer MSGC layers are substituted by layers of silicon micro-strips leading to an allsilicon configuration. The layout of the all-silicon tracker was subsequently optimized in terms of performance and cost, before finally being frozen in the fall of 2001. It was ascertained that the two versions of the tracker provide comparable hit reconstruction quality for tracks with $\mathrm{p}_{\mathrm{T}}>2 \mathrm{GeV} / \mathrm{c}$. The single track reconstruction performance as well as the track reconstruction efficiency in jets are not affected by the change of layout. The pixel detector consists of three barrel layers placed at mean radii of 
4.4, 7.3 and $10.2 \mathrm{~cm}$, from the beam axis and two endcap disks on each side. This detector has been designed to give a two hits per track up to rapidities of about $|\eta|=2.2$ (for tracks originating within $2 \sigma$ around the centre) for large $\mathrm{p}_{\mathrm{T}}$ tracks. The detector occupancy is low due to a small pixel size $150 \mu \mathrm{m} \times 150 \mu \mathrm{m}$. The silicon strip tracker is based on micro-strip silicon devices with a thickness between 320 and $500 \mu \mathrm{m}$ and a pitch size between $\sim 80$ and $\sim 180 \mu \mathrm{m}$. This detector consists of the inner barrel of four layers, the outer barrel of six layers, the inner endcap made of three small disks on each side and the outer endcap made of nine large disks on both sides.

The electromagnetic calorimeter [21] is located between the tracker and the hadron calorimeter, and is composed of about $80000 \mathrm{PbWO}_{4}$ crystals covering the rapidity range up to $|\eta|<3$. Precise energy measurement for photons and electrons can be performed up to $|\eta|<2.5$ except for the region $1.4442<|\eta|<1.5660$. In the ECAL barrel part the crystals are tilted so that their axes make an angle of $3^{\circ}$ with a line from the nominal vertex point. Each of them covers approximately $\Delta \eta \times \Delta \phi$ $=0.0174 \times 0.0174$. The crystal length is $230 \mathrm{~mm}\left(25.8 \mathrm{X}_{0}\right)$. The endcap consists of identically shaped crystals, slightly shorter $\left(220 \mathrm{~mm}, 24.7 \mathrm{X}_{0}\right)$ and somewhat larger in crosssection than the barrel crystals, grouped in mechanical units of $5 \times 5$ crystals (super-crystals). The crystals and super-crystals are arranged in a rectangular $\mathrm{x}$-y grid, with the crystal axes off-pointing from the nominal vertex by angles between $2^{\circ}$ and $5^{\circ}$. A preshower detector, made of lead radiators $\left(3 \mathrm{X}_{0}\right)$ and two orthogonal planes of silicon strip detectors, covers most of the endcap calorimeter $(|\eta|>1.653)$. The energy resolution for a single electromagnetic shower inside the barrel (endcap) measured with a $5 \times 5$ crystal array at low luminosity $\left(10^{33} \mathrm{~cm}^{-2} \mathrm{~s}^{-1}\right)$ is $\sigma_{\mathrm{E}} / \mathrm{E}=2.7(5.7) \% / \sqrt{\mathrm{E}} \oplus 0.155(0.770) \mathrm{MeV} / \mathrm{E} \oplus 0.55 \%(\mathrm{E}$ in $\mathrm{GeV})$ [21]. Different stochastic term for the barrel and endcap is due to the presence of the preshower detector in the endcap while the difference in the noise term is mainly due to the different preamplifier noise.

The sampling hadron calorimeter [22] extends up to $|\eta|=3$, and consists of $4 \mathrm{~mm}$ thick plastic scintillator tiles inserted between brass absorber plates. The barrel and endcap parts of the calorimeter are placed inside the solenoid. The outer hadron calorimeter is located in the central region of the detector, $|\eta|<1.305$, outside the solenoid in the barrel return yoke to measure the late shower development. The calorimeter is more than 10 interaction lengths thick over $|\eta|<3.0$. The lateral granularity of the calorimeter towers is $\Delta \eta \times$ $\Delta \phi=0.087 \times 0.087$ for $|\eta|<2$. The calorimeter readout has a dynamic range from $5 \mathrm{MeV}$ to $3 \mathrm{TeV}$ to allow single muons to be observed. Test beam studies with a full ECAL+HCAL prototype indicate that an energy resolution of $\sigma_{\mathrm{E}} / \mathrm{E}=112 \% / \sqrt{\mathrm{E}} \oplus 3.6 \%(\mathrm{E}$ in $\mathrm{GeV})$ is achievable for single pions with energies between $20 \mathrm{GeV}$ and $300 \mathrm{GeV}$ [22]. To extend the hermeticity of the hadron calorimeter up to $|\eta|=5.191$, a separate forward calorimeter is placed at a distance of $11 \mathrm{~m}$ from the interaction point. This calorimeter, situated in a high radiation and high rate environment, uses quartz fibers as an active medium, embedded in the iron absorber wedges. The forward calorimeter is longitudinally separated into two compartments, an electromagnetic section corresponding to 16 radiation lengths, and a hadronic section corresponding to approximately 9 interaction lengths.

The muon system [23] consists of four stations of muon chambers with excellent time resolution in the central barrel and in the endcap regions and provides a hermetic coverage up to $|\eta|<2.4$. The system is based on drift tubes in the barrel region, cathode strip chambers in the endcap regions and resistive plate chambers in both barrel and endcap chambers. The time-tagging capability of the drift tubes allows bunch crossings to be identified. The cathode strip chambers in the endcap regions are capable to give precise spatial and timing information in the presence of strong magnetic field and large particle rate. The muon system, operating inside the magnetic field return, gives a standalone muon momentum measurement, essential for the first level (Level-1) trigger and useful for the matching between the muon system and the tracker at the High Level Trigger (HLT).

The detector is assumed to be properly calibrated and aligned for the studies presented in this work. For the pixel and silicon strip detectors an alignment with an accuracy of about $10 \mu \mathrm{m}$ in all three dimensions is required. The ultimate momentum resolution for very high $\mathrm{p}_{\mathrm{T}}$ muons $\left(\mathrm{p}_{\mathrm{T}}>200 \mathrm{GeV} / c^{2}\right)$ is reached with a $\sim 100 \mu \mathrm{m}$ alignment of the muon system with respect to the tracker.

To simulate the detector, a GEANT-based detector simulation package CMSIM [24] and a fast detector simulation package CMSJET [25] with parametrizations from full detector simulations were used. In particular a fast tracker response simulation [26] was made on the basis of the full simulation of the silicon-MSGC tracker design [19]. The energy resolution of the electrons, photons and hadrons corresponds to what has been obtained from full simulations in $[21,22]$. Transverse and longitudinal shower development in the calorimeter was simulated as well as effects of the main gaps with dead materials.

\subsection{Trigger}

Detailed descriptions of the Level-1 and HLT triggers can be found in $[27,28]$. Trigger simulations are also presented in detail in these references. The allowed Level-1 trigger bandwidth is $16 \mathrm{kHz}(33 \mathrm{kHz})$ at low (high) luminosity. An equal allocation was assumed across the four categories of objects, taken as (a) electrons/photons, (b) muons, (c) $\tau$ jets and (d) jets, jet-plus-electron, jet-plusmuon and jet-plus- $E_{\mathrm{T}}^{\mathrm{miss}}$. Thresholds for the single and double-object triggers were optimized for a given rate using selected physics channels. For the single and double $\tau$ triggers, the efficiency for the $\mathrm{A} \rightarrow \tau^{+} \tau^{-}$channel versus the efficiency for the $\mathrm{H}^{ \pm} \rightarrow \tau \nu_{\tau}$ channel was used. For the electron/photon trigger, the $\mathrm{W} \rightarrow \mathrm{e} \nu_{\mathrm{e}}$ and $\mathrm{Z} \rightarrow \mathrm{e}^{+} \mathrm{e}^{-}$ channels were considered.

A method of partial event reconstruction is used for the HLT online event selections running in the Filter Farm ex- 
ploiting muon, calorimeter and tracker data to identify the physics objects. Calorimeter, tracker matching and isolation techniques are used to select $\tau$ jets, muons, electrons and photons. Cuts on the $\eta$ separation between two jets are used at the HLT to trigger for invisible Higgs boson decays in the qq $\rightarrow$ qqh process. For a single jet, $b$ tagging with track impact parameter measurement is possible at the HLT. The HLT output rate is $\sim 100 \mathrm{~Hz}$. At low luminosity the $\mathrm{E}_{\mathrm{T}}$ or $\mathrm{p}_{\mathrm{T}}$ thresholds corresponding to a $95 \%$ efficiency are 29 (17) $\mathrm{GeV}$ for single (double) electron; 80 $(40,25) \mathrm{GeV}$ for single (double) photons; 19 (7) GeV/c for single (double) muons (90\% efficiency); 86 (59) GeV for single (double) $\tau$ jets; $180 \mathrm{GeV}$ for jet and $123 \mathrm{GeV}$ for $\mathrm{E}_{\mathrm{T}}^{\text {miss }}$ in the jet-plus- $\mathrm{E}_{\mathrm{T}}^{\text {miss }}$ trigger; $19 \mathrm{GeV}$ for electron and $45 \mathrm{GeV}$ for $\tau$ jet in the electron-plus- $\tau$ jet trigger. For some studies described in this paper somewhat different trigger thresholds were taken. For the $\mathrm{H} \rightarrow \mathrm{ZZ}^{(*)} \rightarrow \ell^{+} \ell^{-} \ell^{\prime+} \ell^{\prime-}$ channel, for example, asymmetrical thresholds of 20 and $15 \mathrm{GeV}$ were taken for the double electron trigger. The $\mathrm{t} \overline{\mathrm{t}} \mathrm{H}, \mathrm{H} \rightarrow \mathrm{b} \overline{\mathrm{b}}$ channel was assumed to be triggered with a single lepton with $\mathrm{p}_{\mathrm{T}} \geq 10 \mathrm{GeV} / \mathrm{c}$. The study is in progress to re-evaluate the discovery potential for $t \bar{t} \mathrm{H}, \mathrm{H} \rightarrow \mathrm{b} \overline{\mathrm{b}}$ channel with the latest trigger table including combined lepton-plus-jet and b-jet triggers. Full trigger simulation is already included in the studies for the channels like the $\mathrm{H} / \mathrm{A} \rightarrow \tau^{+} \tau^{-} \rightarrow$ two jets and the invisible Higgs boson decays from the qq $\rightarrow$ qqh production process.

\subsection{Reconstruction}

\subsubsection{Photons}

A detailed description of the photon and $\mathrm{H} \rightarrow \gamma \gamma$ reconstruction can be found in [21] and [29]. The photon conversions in the tracker material can degrade the Higgs boson mass resolution and can lead to event losses. The studies for the $\mathrm{H} \rightarrow \gamma \gamma$ channel were performed with a tracker design where about $24 \%$ (35\%) of photons convert in the tracker volume in front of the barrel (endcap) ECAL. A matrix of $5 \times 5$ crystals is used to measure the energy of non-converted photons. Dedicated algorithms have been developed to identify and reconstruct the converted photons in the calorimeter. About $6 \%$ of the photon conversions are not recoverable. The effective RMS (the interval containing $68 \%$ events) of the reconstructed Higgs mass at low luminosity for the events without conversions is 0.78 $\mathrm{GeV} / c^{2}$, while for the events with recovered conversions included the effective RMS is $0.87 \mathrm{GeV} / c^{2}$. The hadronic jets misidentified as single photons are first suppressed with isolation cuts in the tracker keeping the photon efficiency at $95 \%$. Rejection of the neutral isolated pions is performed using the lateral shower shape measured in the crystals in the barrel part and the preshower detector in the endcap parts of the calorimeter. For $\mathrm{p}_{\mathrm{T}}^{\pi^{0}}=40 \mathrm{GeV} / c$, for instance, about $70 \%$ of the $\pi^{0}$ 's are rejected in the barrel region and about $55 \%$ in the endcaps for a $90 \%$ efficiency for single photon. The reconstruction and selection efficiency for a single photon from $\mathrm{H} \rightarrow \gamma \gamma$ is estimated to be $74 \%$. The inefficiency consists of losses in the fiducial area of the electromagnetic calorimeter (7.5\%), lost conversions $(6 \%)$, losses due to the isolation requirements (5\%) and losses due to the $\pi^{0}$ rejection (10\%). The events in which a Higgs boson is produced contain more large$\mathrm{p}_{\mathrm{T}}$ tracks than minimum-bias pileup events. This feature is exploited to find the vertex for the $\mathrm{H} \rightarrow \gamma \gamma$ mass reconstruction. The efficiency and the purity of this method depends in particular on the minimum-bias event model and on the $\mathrm{p}_{\mathrm{T}}$ spectrum of the Higgs boson as well as on the luminosity. The Higgs boson mass resolution ( $\sigma$ of the gaussian fit) is $0.65(0.69) \mathrm{GeV} / c^{2}$ at low (high) instantaneous luminosity for $\mathrm{m}_{\mathrm{H}}=100 \mathrm{GeV} / \mathrm{c}^{2}$. A mass window of $\pm 1.4 \sigma$ contains $73 \%$ of events at low luminosity and $69 \%$ at high luminosity.

\subsubsection{Electrons}

An electron is identified as an ECAL cluster associated with a track in the inner detector. Before reaching the ECAL, electrons radiate in the material between the interaction point and the ECAL. For example, for electrons with $\mathrm{p}_{\mathrm{T}}=35 \mathrm{GeV} / c$ and $|\eta| \leq 1.5$, the mean energy loss is $43.6 \%$ before exiting the tracker volume [30] in the tracker design described in [20]. This corresponds to an average $0.57 \mathrm{X}_{0}$ of material. Most of the energy is radiated as quasi-collinear low energy photons. The bending of the electron in the $4 \mathrm{~T}$ magnetic field results in a spray of energy reaching the ECAL. The distribution of this energy is, to good approximation, only in the $\varphi$ direction. Bremsstrahlung recovery algorithms, based on an asymmetrical crystal window [31] or on more dedicated clustering methods $[30,32]$, have been developed. The energy resolution for electrons with $\mathrm{p}_{\mathrm{T}}=35 \mathrm{GeV} / \mathrm{c}$ in the barrel is $1.2(1.5) \%$ for low (high) luminosity [28]. The angular resolution in $\varphi$ is $1.7(1.9) \mathrm{mrad}$ and in $\eta$ it is $1.1 \times 10^{-3}$. The electron reconstruction efficiency in the tracker for $\mathrm{p}_{\mathrm{T}}^{\mathrm{e}} \geq 10 \mathrm{GeV} / \mathrm{c}$ is greater than $90 \%$ even in the $\eta$ regions with the largest number of $\mathrm{X}_{0}$ [19]. An algorithm for electron momentum measurement in the tracker taking into account bremsstrahlung energy losses is under development [33]. The $\mathrm{H} \rightarrow \mathrm{ZZ}^{*} \rightarrow \mathrm{e}^{+} \mathrm{e}^{-} \mathrm{e}^{+} \mathrm{e}^{-}$channel was studied in $[32,34,35]$ using somewhat different reconstruction algorithms and assumptions for the ECAL energy resolution and tracker design. The Higgs boson mass resolution, still with the silicon-MSGC tracker version, was found at low luminosity to be $1.3,1.6$ and $1.8 \mathrm{GeV} / \mathrm{c}^{2}$ for $\mathrm{m}_{\mathrm{H}}=130$, $150,170 \mathrm{GeV} / \mathrm{c}^{2}$, respectively, and the $67 \%$ of events were found within $\pm 2 \sigma$ [32]. Studies with the latest tracker design with about $30 \%$ more material are in progress.

\subsubsection{Muons}

The muon $\mathrm{p}_{\mathrm{T}}$ is measured with a precision of $\sim 1 \%$ up to $\mathrm{p}_{\mathrm{T}} \sim 100 \mathrm{GeV} / \mathrm{c}$ in the barrel part of the tracker. The average Level-1 plus HLT reconstruction efficiency is $97 \%$ for muons within $5<\mathrm{p}_{\mathrm{T}}<100 \mathrm{GeV} / \mathrm{c}$ and $|\eta|<2.1$ [28]. The discovery potential for the $\mathrm{H} \rightarrow \mathrm{ZZ}^{(*)} \rightarrow \ell^{+} \ell^{-} \ell^{\prime+} \ell^{\prime-}$ 
channel in $[34,36]$ was evaluated with an earlier tracker version. The Higgs boson mass resolution in the four-muon decay mode was found to be 0.8 and $0.9 \mathrm{GeV} / \mathrm{c}^{2}$ for $\mathrm{m}_{\mathrm{H}}$ $=130$ and $150 \mathrm{GeV} / \mathrm{c}^{2}$, respectively $[23,36]$. These resolutions are somewhat better than those from the preliminary simulations with the present tracker design. The single muon reconstruction efficiency was taken to be $90 \%$, which is lower than that of [28]. Due to the compensation of these two factors no sizeable change is expected for the discovery potential for the $\mathrm{H} \rightarrow \mathrm{ZZ}^{(*)} \rightarrow \mu^{+} \mu^{-} \mu^{+} \mu^{-}$and $\mathrm{H} / \mathrm{A} \rightarrow \mu^{+} \mu^{-}$channels.

\subsubsection{Jets}

Reconstruction of hadronic jets is performed with an iterative cone algorithm [37], with a cone size of 0.5 , and taking as input the energy and $(\eta, \varphi)$ position of the calorimeter towers. Jet energy corrections take into account the nonlinear response to hadrons, out-of-cone energy leakage due to magnetic field and showering and energy offset due to pile up and one-sided zero-suppression (only those channels that are above a threshold are read out). Energy corrections are derived from Monte Carlo data by comparing the energy of jets obtained from the generator-level particles in the hard interaction to that of jets reconstructed in the calorimeter with the same cone size. In the latest simulations [28] the stochastic and constant terms of the jet transverse energy resolution for low luminosity are 1.18 and 0.07 . This resolution is $\sim 20 \%$ worse than those used for some studies in this paper, in particular for the study of the $t \bar{t} \mathrm{H}, \mathrm{H} \rightarrow \mathrm{b} \bar{b}$ channel. However, further improvements are expected for the jet resolution and energy scale from the jet energy corrections with charged particles [38]. For many channels, in particular for the gauge boson fusion channels, a central jet veto was used to suppress backgrounds. An $\mathrm{E}_{\mathrm{T}}$ threshold of $\sim 20 \mathrm{GeV}$ can be used for these jets at low luminosity. At high luminosity $\left(10^{34} \mathrm{~cm}^{2} \mathrm{~s}^{-1}\right)$ the full simulation studies [39] show that a jet initiated by a parton with $\mathrm{p}_{\mathrm{T}}=20 \mathrm{GeV} / c$ can be found in the calorimeter with an efficiency of $\sim 70 \%$ with an algorithm where the pile-up energy from on the average of 17.3 minimum bias interactions is subtracted on an event-by-event basis. Studies of the false jet suppression with the usage of the tracker are in progress.

\subsubsection{Missing transverse energy}

Measurement of the missing transverse energy $\left(\mathrm{E}_{\mathrm{T}}^{\mathrm{miss}}\right)$ is affected by the non-linear calorimeter response. A method to improve the $\mathrm{E}_{\mathrm{T}}^{\mathrm{miss}}$ scale using jet energy corrections was developed in [40]. A significant improvement was obtained for the Higgs boson mass reconstruction efficiency in the $\mathrm{H} \rightarrow \tau^{+} \tau^{-}$channel. Despite the escaping neutrinos, the Higgs boson mass can be reconstructed in the $\mathrm{H} \rightarrow \tau^{+} \tau^{-}$ channels from the visible $\tau$ momenta (leptons or $\tau$ jets) and $\mathrm{E}_{\mathrm{T}}^{\mathrm{miss}}$ with the collinearity approximation for the neutrinos from highly boosted $\tau$ 's. The correction of the missing $\mathrm{E}_{\mathrm{T}}$ scale improves the reconstruction efficiency by reducing the number of events with negative reconstructed

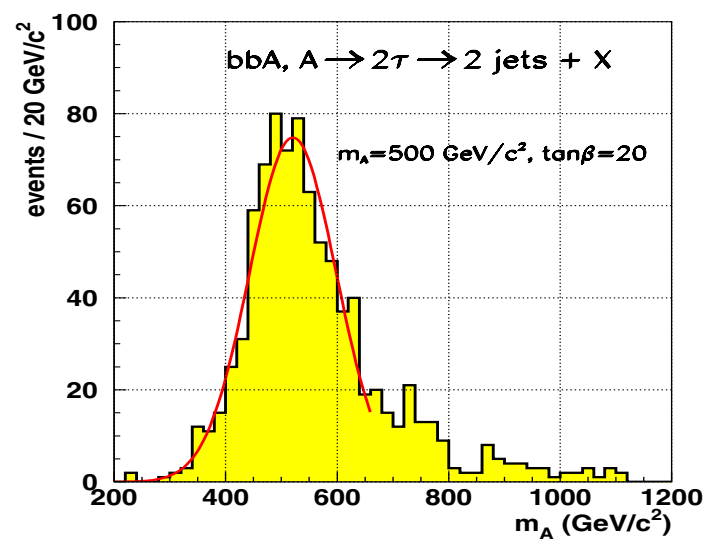

Fig. 5. Higgs boson mass reconstructed with full simulation for the $\mathrm{H}, \mathrm{A} \rightarrow \tau^{+} \tau^{-} \rightarrow$ two $\tau$ jets channel for $\mathrm{m}_{\mathrm{A}}=500 \mathrm{GeV} / c^{2}$, $\tan \beta=20$ at low luminosity

$\tau$ energies. The mass resolution depends on the angle $\Delta \varphi$ between the visible $\tau$ momenta as $1 / \sin (\Delta \varphi)$ and is sensitive to the $\mathrm{E}_{\mathrm{T}}^{\mathrm{miss}}$ measurement, both in magnitude and particularly in direction. Figure 5 [40] shows the Higgs boson mass reconstructed with full simulation for the fully hadronic final state from $\mathrm{H} \rightarrow \tau^{+} \tau^{-} \rightarrow$ two $\tau$ jets for $\mathrm{m}_{\mathrm{A}}=500 \mathrm{GeV} / c^{2}$ with the jet energy corrections. The reconstruction efficiency (including the cut $\Delta \varphi<175^{\circ}$ ) is around $35 \%$ for $\mathrm{m}_{\mathrm{A}}=200$ and $500 \mathrm{GeV} / \mathrm{c}^{2}$. This efficiency, obtained with an $\mathrm{E}_{\mathrm{T}}^{\mathrm{miss}}$ cut, is improved by a factor of about two when a b jet is required in the event, mainly due to a more efficient $\Delta \varphi$ cut [15]. A mass resolution around $15 \%$ is obtained for $\mathrm{m}_{\mathrm{A}}=200$ and $500 \mathrm{GeV} / c^{2}$ with the cut $\Delta \varphi<175^{\circ}$. Energy-flow algorithms are expected to further improve the $\mathrm{E}_{\mathrm{T}}^{\mathrm{miss}}$ measurement.

\subsection{6 $\tau$ identification}

The $\tau$-jet identification takes advantage of the $\tau$-jet properties such as narrowness and low multiplicity. A hadronic $\tau$ decay produces an energy deposit in the calorimeter narrower than that from an average hadronic jet. On average, $85 \%$ of the $\tau$-jet energy is contained in a $3 \times 3$ trigger cell corresponding to a cone size of about 0.1 and $98 \%$ in a cone of 0.4 for $\mathrm{E}_{\mathrm{T}}^{\tau}$ jet $>40 \mathrm{GeV}[41,42]$. These features are used in the Level-1 trigger [28] where transverse profiles of the active tower patterns are analyzed to tag narrow jets as potential hadronic $\tau$ decays. A $\tau$ jet leads to a localized energy deposit in the electromagnetic calorimeter [42-44], which can be used in the Level-2 trigger to confirm the Level-1 $\tau$-jet candidates. In the HLT algorithms, loose isolation criteria are used in the tracker $[45,46]$. At this level, no requirement is made on the number of tracks in the signal cone, to keep one-, three- (and five-) prong $\tau$ decays. Offline $\tau$-jet selections are based on a cut on the $\mathrm{p}_{\mathrm{T}}$ of the leading charged particle, the number of charged particles in the signal cone and a signal cone size $(\Delta \mathrm{r}<$ 0.04) smaller than that used at the HLT. In the study of the $\mathrm{H}, \mathrm{A} \rightarrow \tau^{+} \tau^{-}$channel with lepton-plus- $\tau$-jet final state [47], one prong hadronic $\tau$ decays were selected with 


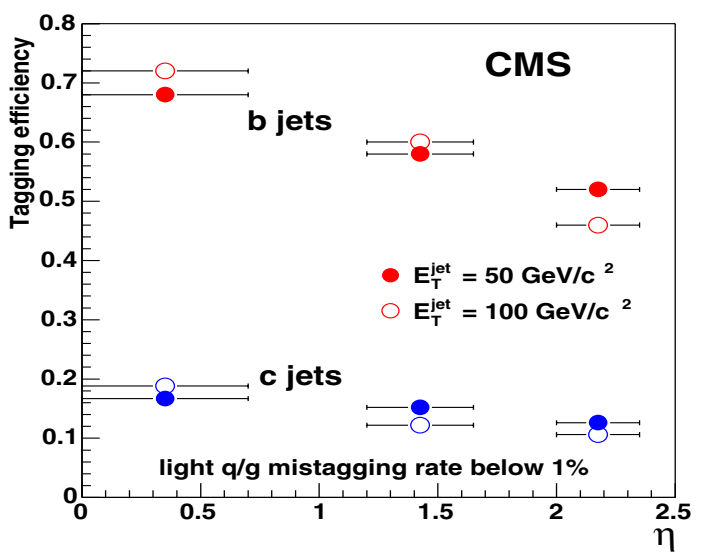

Fig. 6. Tagging efficiencies for $b$ and $c$ jets with $\mathrm{E}_{\mathrm{T}}^{\mathrm{jet}}=50$ and $100 \mathrm{GeV}$ for a $10^{-2}$ mistagging rate for light quark jets. A track counting method with impact parameter measurement is used

$\mathrm{p}_{\mathrm{T}}>10 \mathrm{GeV} / c$ for the single track in the $\tau$ jet. The $\tau$-jet selection efficiency in this channel for $\mathrm{E}_{\mathrm{T}}^{\mathrm{jet}}>40 \mathrm{GeV}$ was found to be $0.32(0.36)$ for $\mathrm{m}_{\mathrm{A}}=140(300) \mathrm{GeV} / \mathrm{c}^{2}$ and the misidentification efficiency for the jets from the $\mathrm{W}+$ jet background $2 \times 10^{-3}$ [43]. In the study of the two- $\tau$-jet final state from the $\mathrm{H}, \mathrm{A} \rightarrow \tau^{+} \tau^{-}$channel [15], a high momentum particle with $\mathrm{p}_{\mathrm{T}} \geq 40 \mathrm{GeV} / c$ was required in the core of the jet and for the signal cone around the leading charged particle direction $\Delta \mathrm{r}=0.03$ - 0.04 was taken $(\Delta \mathrm{r}=0.07$ at the trigger level). The track isolation was performed in the area limited by the signal cone and the isolation cone $(\Delta \mathrm{r}=0.4)$. If one or three charged particles are required in the signal cone the efficiency of the $\tau$-jet selection, including the Level-1 and the HLT trigger efficiency, was found to be $0.8 \%$ and $9 \%$ for $\mathrm{m}_{\mathrm{A}}=200$ and 500 $\mathrm{GeV} / c^{2}$, respectively. A rejection factor of about 1000 per jet was obtained. Highly energetic hadronic jets can be further suppressed with an additional cut in $\mathrm{p}_{\mathrm{T}}^{\text {track }} / \mathrm{E}_{\mathrm{T}}^{\mathrm{jet}}$ with a small loss of signal efficiency. The $\tau$ lifetime can be exploited with the impact parameter measurement to reduce the $\mathrm{Z} \rightarrow \ell^{+} \ell^{-}$background to $\mathrm{H}, \mathrm{A} \rightarrow \tau^{+} \tau^{-} \rightarrow \ell^{+} \ell^{-}+\mathrm{X}$ and to further reduce the QCD multi-jet background for $\mathrm{H}, \mathrm{A} \rightarrow \tau^{+} \tau^{-} \rightarrow$ two $\tau$ jets $+\mathrm{X}[48]$. A rejection at least 9 was obtained against the QCD multi-jet background with an efficiency of $\sim 60 \%$ for the $\mathrm{H}, \mathrm{A} \rightarrow \tau^{+} \tau^{-}$events when the charge correlation between the $\tau$ jets was also included [15]. For the tree-prong $\tau$ decays, secondary vertex reconstruction can be used to reduce more efficiently the hadronic jet background.

The energy scale for $\tau$ jets could be evaluated with $\gamma$-plus-jet events used for the energy calibration of the "normal" jets. The statistical precision, however is going to be $\simeq 30$ times worse than for the "normal" jets, since jets should pass the $\tau$-identification criteria.

\subsubsection{Tagging of $b$ jets}

The b-jet tagging is performed with the reconstruction of the secondary vertices or with impact parameter measure- ment. The precision of the impact parameter measurement in the transverse plane is $\sim 10 \mu \mathrm{m}$ for $\mathrm{p}_{\mathrm{T}}=100 \mathrm{GeV} / c$ and $\sim 100 \mu \mathrm{m}$ for $\mathrm{p}_{\mathrm{T}}=1 \mathrm{GeV} / c$. Unless stated otherwise, the tagging probability is calculated by requiring two tracks with $\mathrm{p}_{\mathrm{T}}>1 \mathrm{GeV} / c$ and $\sigma_{\mathrm{ip}}>2$ inside the jet cone, where $\sigma_{\mathrm{ip}}$ is the significance of the track impact parameter in the transverse plane. Efficiencies to tag $\mathrm{b}$ and $\mathrm{c}$ jets for $\mathrm{E}_{\mathrm{T}}^{\mathrm{jet}}=50$ and $100 \mathrm{GeV}$ with a $1 \%$ mistagging probability for light quark and gluon jets are shown in Fig. 6 as a function of $\left|\eta^{\text {jet }}\right|$ from a full simulation and complete reconstruction study with the final tracker design [49]. The efficiency to tag b jets, $\simeq 70 \%$ ( $\simeq 50 \%$ ) for the barrel (endcap) part of the tracker is about $20 \%$ higher than that used for the studies in this paper.

\subsection{Signal and background simulation}

The PYTHIA Monte-Carlo program [50] was used to generate signal and background events unless stated otherwise. The HERWIG [51] generator was used for the Higgs boson production from gaugino decays and in the study of charged Higgs bosons decaying to gauginos. The MSSM sparticle spectrum and decay branching fractions were calculated with ISASUSY 7.58 [52] (interfaced to HERWIG). The CompHEP program [53] was used to generate higherorder background processes such as: $t \overline{t j j}$ and $t \bar{t} b \bar{b}, t \overline{\mathrm{j} j}$, electroweak Zjj and Wjj. The CompHEP program was interfaced with PYTHIA for fragmentation and hadronization [54]. The QCD Zjj and Wjj backgrounds were generated with the programs based on the work of [55] and interfaced to PYTHIA. The signal and all backgrounds for the exclusive $\mathrm{H} \rightarrow \gamma \gamma$ channels in the $\mathrm{H}+$ jet, $\mathrm{qq} \rightarrow \mathrm{qqH}$ and $\mathrm{WH}$ production processes were generated with CompHEP. The evaluation of the $\gamma$-plus-three-jet background is in progress with CompHEP.

The helicity correlations in the $\mathrm{H}^{ \pm} \rightarrow \tau \nu_{\tau}$ and $\mathrm{W}^{ \pm} \rightarrow$ $\tau \nu_{\tau}$ decays were accounted for by the TAUOLA package [56]. The production cross sections and branching fractions for the two- $\tau$-jet and two-lepton final states from the $\mathrm{gg} \rightarrow \mathrm{b} \overline{\mathrm{b}} \mathrm{H} / \mathrm{A}, \mathrm{H} / \mathrm{A} \rightarrow \tau^{+} \tau^{-}$processes and the branching fractions for the $\mathrm{gb} \rightarrow \mathrm{tH}^{ \pm}, \mathrm{H}^{ \pm} \rightarrow \tau \nu_{\tau}$ channel were calculated with the programs of $[6,12]$. The LO production cross section for the $\mathrm{gb} \rightarrow \mathrm{tH}^{ \pm}$process was taken from [7]. The cross sections and branching fractions for the lighter scalar MSSM Higgs boson were also calculated with the programs of $[6,12]$.

Simple sequential cut-based analysis were used. The expected statistical significance was calculated using Poisson statistics for the channels where backgrounds are below $\sim 20$ events and approximated otherwise by $\mathrm{N}_{\mathrm{S}} / \sqrt{\mathrm{N}_{\mathrm{B}}}$, where $\mathrm{N}_{\mathrm{S}}$ and $\mathrm{N}_{\mathrm{B}}$ are the numbers of the signal and background events, respectively. Systematic uncertainties on the background evaluations were not taken into account in the calculation of the statistical significance. A combined statistical significance was calculated as a quadratic sum of the individual significance. The NLO production cross sections were used for both signal and backgrounds in the inclusive $\mathrm{H} \rightarrow \gamma \gamma$ and $\mathrm{H} \rightarrow \gamma \gamma+$ jet channels and in the $\mathrm{H} \rightarrow \mathrm{ZZ}^{*} / \mathrm{ZZ} \rightarrow \ell^{+} \ell^{-} \ell^{\prime+} \ell^{\prime-}$ and $\mathrm{H} \rightarrow \mathrm{WW}^{*} / \mathrm{WW} \rightarrow$ 
$\ell \ell \nu_{\ell} \nu_{\ell}$ channels with the $\mathrm{k}$ factors given in the following sections. Detailed information on the cross sections and $\mathrm{k}$ factors can be found in [21] for the inclusive $\mathrm{H} \rightarrow \gamma \gamma$ channel, in [29] for the $\mathrm{H} \rightarrow \mathrm{ZZ}^{*} / \mathrm{ZZ} \rightarrow \ell^{+} \ell^{-} \ell^{\prime+} \ell^{\prime-}$ channel and in [57] for the $\mathrm{H} \rightarrow \mathrm{WW}^{*} / \mathrm{WW} \rightarrow \ell \ell \nu_{\ell} \nu_{\ell}$ channel.

\section{Standard searches}

\subsection{Searches for a SM-like scalar Higgs boson H}

The four-lepton channel from $\mathrm{H} \rightarrow \mathrm{ZZ}^{*} \rightarrow \ell^{+} \ell^{-} \ell^{\prime+} \ell^{\prime-}$ was investigated in [34] with fast simulation. Backgrounds from the $\mathrm{ZZ}^{*}$, $\mathrm{t} \overline{\mathrm{t}}$ and $\mathrm{Zb} \overline{\mathrm{b}}$ processes were suppressed by requiring the leptons to be isolated in the tracker, an upper bound on the lepton impact parameter significance and with cuts on the two-lepton and four-lepton invariant masses. For the largest $\mathrm{p}_{\mathrm{T}}$ electron or muon, $\mathrm{p}_{\mathrm{T}}>20 \mathrm{GeV} / c$ was required, the threshold for the secondlargest-p $\mathrm{p}_{\mathrm{T}}$ electron (muon) was taken to be $15(10) \mathrm{GeV} / c$ and the thresholds for the other two electrons (muons) were taken to be $10(5) \mathrm{GeV} / c$. For isolated leptons within $\left|\eta^{\ell}\right|<2.5$ these thresholds lead to a four-electron acceptance of $33 \%$ and a four-muon acceptance of $41 \%$ for $\mathrm{m}_{\mathrm{H}}=130-150 \mathrm{GeV} / c^{2}$. The four-electron final state was studied with full simulation and complete reconstruction [32]. The reconstructed four-electron invariant mass distribution of the $\mathrm{H} \rightarrow \mathrm{ZZ}^{*} \rightarrow \mathrm{e}^{+} \mathrm{e}^{-} \mathrm{e}^{+} \mathrm{e}^{-}$signal and the background with $\mathrm{m}_{\mathrm{H}}=130,150$ and $170 \mathrm{GeV} / c^{2}$ for $100 \mathrm{fb}^{-1}$ is shown in Fig. 7. For the calculation of the statistical significance for $\mathrm{H} \rightarrow \mathrm{ZZ}^{*} / \mathrm{ZZ} \rightarrow \ell^{+} \ell^{-} \ell^{\prime+} \ell^{\prime-}$ the results of [34] were used. The CTEQ2L structure functions were used in PYTHIA. The gg $\rightarrow \mathrm{H}$ and $\mathrm{qq} \rightarrow \mathrm{qqH}$ componenets of the signal cross section were normalized to the NLO cross sections of [58] calculated with the MRS(R1) structure functions. The average $\mathrm{k}$ factor for the signal is therefore $2.25-1.56$ for the mass region of $130 \leq \mathrm{m}_{\mathrm{H}} \leq 600 \mathrm{GeV} / c^{2}$. For the qq $\rightarrow \mathrm{ZZ}^{*}$ background the $\mathrm{k}$ factor was taken to be 1.2 and $1.2-1.47$ for the $\mathrm{qq} \rightarrow \mathrm{ZZ}$ process in the region $190 \leq \mathrm{m}_{\mathrm{ZZ}} \leq 600 \mathrm{GeV} / c^{2}$. The $\mathrm{gg} \rightarrow \mathrm{ZZ}$ component was taken into account multiplying the $\mathrm{LO}$ qq $\rightarrow \mathrm{ZZ}$ process by a factor of 1.33. The $t \bar{t}$ background was normalized to the NLO cross section of $760 \mathrm{pb}$ ( $\mathrm{k}$ factor 1.27). No k factor was used for the Z $\mathrm{b} \overline{\mathrm{b}}$ background.

Around $\mathrm{m}_{\mathrm{H}} \sim 170 \mathrm{GeV} / c^{2}$, where the $\mathrm{H} \rightarrow$ $\mathrm{ZZ}^{*} / \mathrm{ZZ}$ branching fraction is smallest, the $\mathrm{H} \rightarrow$ $\mathrm{WW}^{*} / \mathrm{WW} \rightarrow \ell^{+} \nu_{\ell} \ell^{-} \nu_{\ell}$ channel was exploited [57,59]. For $\mathrm{m}_{\mathrm{H}} \lesssim 200 \mathrm{GeV} / c^{2}$, the backgrounds from the $\mathrm{WW}$, $\mathrm{t} \overline{\mathrm{t}}$ and $\mathrm{Wt}$ processes were suppressed by taking advantage of WW spin correlations, which turn into small $\ell^{+} \ell^{-}$ opening angles. For $\mathrm{m}_{\mathrm{H}} \gtrsim 200 \mathrm{GeV} / c^{2}$, a cut in the transverse momentum of the lepton-pair was used. Central-jet vetoing was used to suppress further the $t \bar{t}$ and Wt backgrounds. Figure 8 shows the distribution of the $\mathrm{p}_{\mathrm{T}}$ of the smaller-p $\mathrm{p}_{\mathrm{T}}$ lepton of the $\mathrm{H} \rightarrow \mathrm{WW}^{*} \rightarrow \ell^{+} \nu_{\ell} \ell^{-} \nu_{\ell}$ signal, the total background and the WW background for a 30$45 \mathrm{GeV} / c$ window in the $\mathrm{p}_{\mathrm{T}}$ of the larger $\mathrm{p}_{\mathrm{T}}$ lepton with $\mathrm{m}_{\mathrm{H}}=140 \mathrm{GeV} / c^{2}$ for $30 \mathrm{fb}^{-1}$. The MRS(A) structure functions were used in PYTHIA. As for $\mathrm{H} \rightarrow \mathrm{ZZ}^{*} / \mathrm{ZZ} \rightarrow$ $\ell^{+} \ell^{-} \ell^{\prime+} \ell^{\prime-}$, the $\mathrm{gg} \rightarrow \mathrm{H}$ and $\mathrm{qq} \rightarrow \mathrm{qqH}$ componenets of

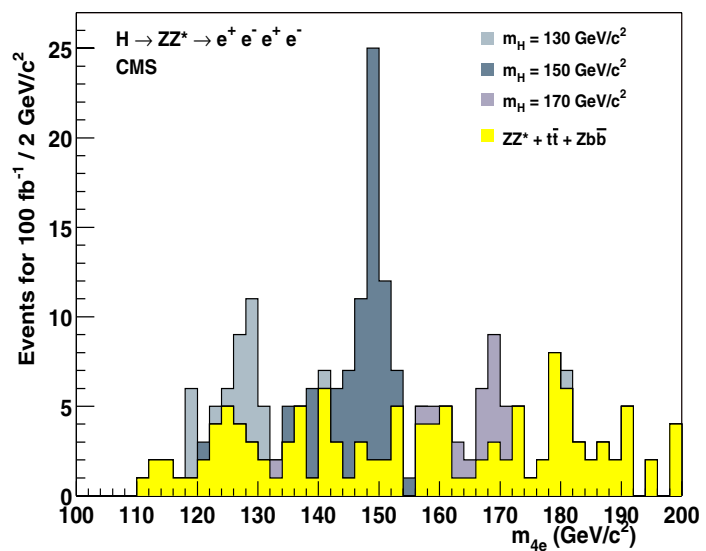

Fig. 7. Reconstructed four-electron invariant mass distribution of the $\mathrm{H} \rightarrow \mathrm{ZZ}^{*} \rightarrow \mathrm{e}^{+} \mathrm{e}^{-} \mathrm{e}^{+} \mathrm{e}^{-}$signal (dark) and the background (light) with $\mathrm{m}_{\mathrm{H}}=130,150$ and $170 \mathrm{GeV} / c^{2}$ for $100 \mathrm{fb}^{-1}$ with $\mathrm{k}$ factors for both signal and background

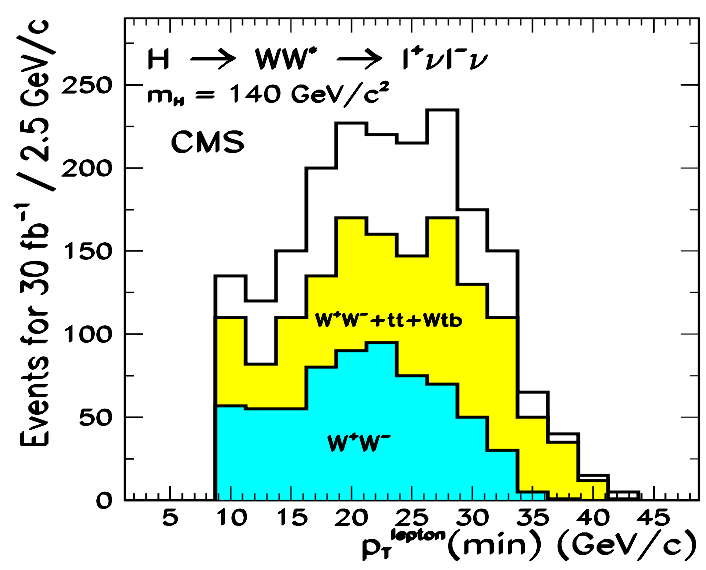

Fig. 8. The $\mathrm{p}_{\mathrm{T}}$ distribution of the smaller $\mathrm{p}_{\mathrm{T}}$ lepton of the $\mathrm{H} \rightarrow \mathrm{WW}^{*} \rightarrow \ell^{+} \nu_{\ell} \ell^{-} \nu_{\ell}$ signal (white), in the total background (light) and in the $\mathrm{W}^{+} \mathrm{W}^{-}$background (dark) with $\mathrm{m}_{\mathrm{H}}$ $=140 \mathrm{GeV} / c^{2}$ for $30 \mathrm{fb}^{-1}$ with $\mathrm{k}$ factors for both signal and background

the signal cross section were normalized to the NLO cross sections of [58] calculated with the MRS(R1) structure functions. The resulting average $\mathrm{k}$ factor for the signal is $1.7-1.5$ for the mass region of $120 \leq \mathrm{m}_{\mathrm{H}} \leq 500 \mathrm{GeV} / c^{2}$. For the WW background the $\mathrm{k}$ factor was taken to be 1.5, for $t \bar{t}$ background 1.16 and no $\mathrm{k}$ factor was used for the Wt background. The LO cross section times branching fraction for the Wt process was taken to be $6 \mathrm{pb}$ [57].

Figure 9 shows the reconstructed di-photon invariant mass distribution of the inclusive $\mathrm{H} \rightarrow \gamma \gamma$ signal and the background with $\mathrm{m}_{\mathrm{H}}=130 \mathrm{GeV} / \mathrm{c}^{2}$ for $100 \mathrm{fb}^{-1}$. The background includes the irreducible direct $\mathrm{pp} \rightarrow \gamma \gamma+\mathrm{X}$ production and the $\mathrm{pp} \rightarrow \gamma+$ jet $+\mathrm{X}$ production with a hard bremsstrahlung from a quark jet [21]. The QCD multi-jet background and the $\gamma$-plus-jet background with the jet fragmenting into a leading isolated $\pi^{0}$ were not included. Preliminary full simulation studies indicate that these backgrounds are below $40 \%$ of the irreducible backgrounds. In this inclusive search the signal-to-background 


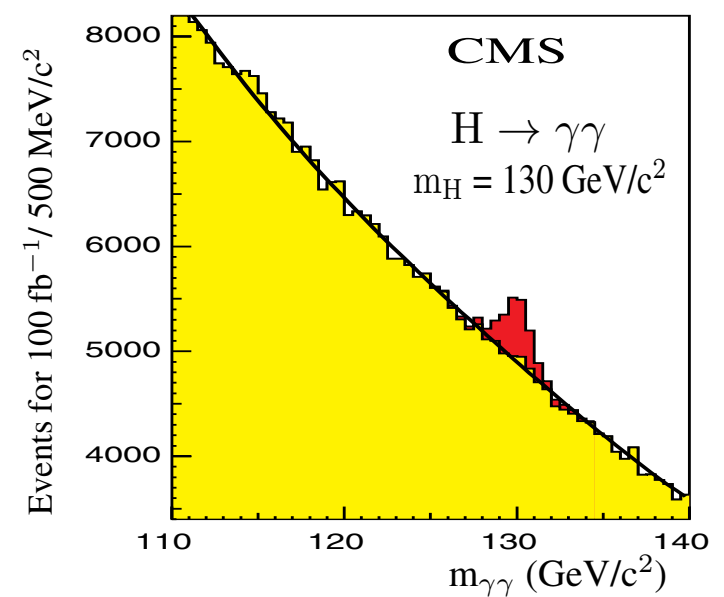

Fig. 9. Reconstructed di-photon invariant mass distribution of the $\mathrm{H} \rightarrow \gamma \gamma$ signal (dark) and the background (light) with $\mathrm{m}_{\mathrm{H}}=130 \mathrm{GeV} / \mathrm{c}^{2}$ for $100 \mathrm{fb}^{-1}$ with $\mathrm{k}$ factors for both signal and background

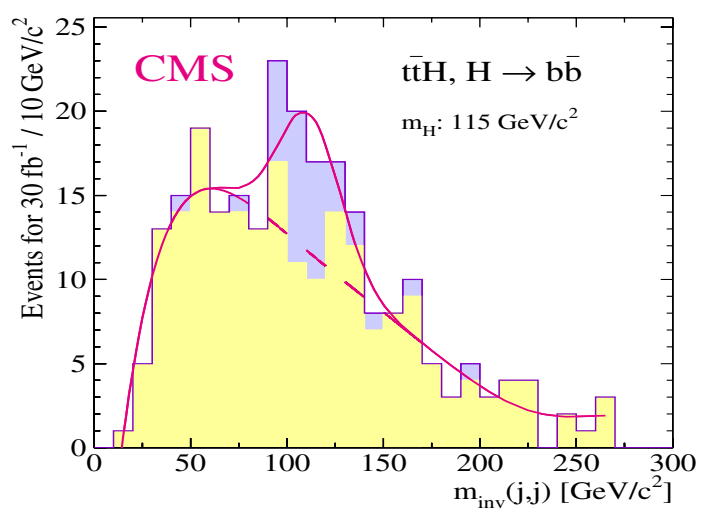

Fig. 10. Jet-jet invariant mass distribution of the $\mathrm{t} \overline{\mathrm{t}} \mathrm{H} \rightarrow$ $\ell^{ \pm} \nu_{\ell} \mathrm{q} \overline{\mathrm{q}} \mathrm{b} \overline{\mathrm{b}} \mathrm{b} \overline{\mathrm{b}}$ signal (dark) and the background (light) with $\mathrm{m}_{\mathrm{H}}=115 \mathrm{GeV} / c^{2}$ for $30 \mathrm{fb}^{-1}$

ratio is of the order 1/10. In Fig. 9, $\mathrm{k}$ factors are used for the signal and for the backgrounds. The CTEQ4M structure functions were used in PYTHIA. The gg $\rightarrow \mathrm{H}$ and $\mathrm{qq} \rightarrow \mathrm{qqH}$ components of the signal cross section of PYTHIA were normalized to the NLO cross sections of [58] calculated also with CTEQ4M structure functions. This normalization yields a $\mathrm{k}$ factor of 1.85 - 1.65 for $\mathrm{gg} \rightarrow \mathrm{H}$ and $0.89-0.93$ for $\mathrm{qq} \rightarrow \mathrm{qqH}$ in the mass region of $100 \leq \mathrm{m}_{\mathrm{H}} \leq 150 \mathrm{GeV} / c^{2}$. For the backgrounds, the $\mathrm{k}$ factor for the gg $\rightarrow \gamma \gamma$ process was taken the same as for the $\mathrm{gg} \rightarrow \mathrm{H}$ while no $\mathrm{k}$ factor was used for the qq $\rightarrow \gamma \gamma$ process and for the hard bremsstrahlung process from a quark jet. The statistical significance for $\mathrm{m}_{\mathrm{H}}=100 \mathrm{GeV} / c^{2}$ with $30 \mathrm{fb}^{-1}$ was found to be 3.1 with the LO cross sections and 5.0 with the NLO cross sections with the above $\mathrm{k}$ factors. The $\mathrm{H} \rightarrow \gamma \gamma$ decay channel was studied in [60] in the exclusive production processes, $\mathrm{pp} \rightarrow \mathrm{H}+$ jet $+\mathrm{X}$, where the Higgs boson is produced at large $\mathrm{p}_{\mathrm{T}}$, and in the associated $\mathrm{WH}$ production channel with one isolated lepton from $\mathrm{W} \rightarrow \ell \nu_{\ell}$. Due to an efficient background suppression for these channels, a two-photon mass window of
$3 \mathrm{GeV} / c^{2}$ was taken instead of the $\pm 1.4 \sigma\left(1.9 \mathrm{GeV} / c^{2}\right)$ window used for the inclusive $\mathrm{H} \rightarrow \gamma \gamma$ search. As the gg $\rightarrow \gamma \gamma \mathrm{g}$ component of the pp $\rightarrow \gamma \gamma+$ jet background has been found to be small [61], it was not included in the study of [60]. To further suppress the backgrounds for the $\mathrm{pp} \rightarrow \mathrm{H}+$ jet $+\mathrm{X}$ channel, a reconstructed partonic centre-of-mass energy in excess of $300 \mathrm{GeV}$ was required in addition to the jet requirements. The NLO k factor for the signal and for the background was taken to be 1.6. The $t \bar{t} H$ production channel represents about $35 \%$ of the total $\gamma \gamma+$ lepton production but, to include this component, a complete calculation of the irreducible background from $\mathrm{t} \overline{\mathrm{t}} \gamma \gamma$ is needed.

Figure 10 shows the invariant mass distribution reconstructed from two b jets in the $\mathrm{t} \overline{\mathrm{t}} \mathrm{H} \rightarrow \ell^{ \pm} \nu_{\ell} \mathrm{q} \overline{\mathrm{q}} \mathrm{b} \overline{\mathrm{b}} \overline{\mathrm{b}}$ signal and in the background with $\mathrm{m}_{\mathrm{H}}=115 \mathrm{GeV} / c^{2}$ for $30 \mathrm{fb}^{-1}$ [62]. The background includes the $\mathrm{t} \overline{\mathrm{t}}+\mathrm{jets}, \mathrm{t} \overline{\mathrm{t}} \mathrm{b} \overline{\mathrm{b}}$ and $\mathrm{W}+$ jets processes. The correct event configuration among all possible combinations was first searched for to reconstruct the Higgs boson mass. The best configuration was defined as the one giving the highest value for an event likelihood function including b-tagging efficiencies for four jets, probabilities for two jets to be non-b jets, W mass reconstructions and reconstruction of one hadronically and one leptonically decaying top quark. The backgrounds were suppressed with cuts in the likelihood functions for resonances, b tagging and kinematics. Angular correlations between one of the top quarks and the reconstructed Higgs boson were used in [63] to further reduce the $t \bar{t} b \bar{b}$ background. Fully hadronic final states from the $\mathrm{t} \overline{\mathrm{t}} \mathrm{H} \rightarrow \mathrm{q} \overline{\mathrm{q}} \mathrm{qqb} \overline{\mathrm{b}} \mathrm{b} \overline{\mathrm{b}}$ channel would roughly double the number of $t \bar{t} \mathrm{H}$ signal events but are not yet included. These final states could be triggered with a b-jet trigger combined with multi-jet triggers [64]. To study this channel an evaluation of the higher order QCD multi-jet (eight jets) background is still needed. The WH $\rightarrow \ell^{ \pm} \nu_{\ell} \mathrm{b} \overline{\mathrm{b}}$ channel was studied only for the high luminosity scenario due to the small event rate and many large backgrounds $\left(\mathrm{W}^{ \pm} \mathrm{jj}\right.$, WZ, WtE, tb) [62].

The gauge boson fusion production was studied in the $\mathrm{H} \rightarrow \gamma \gamma, \mathrm{H} \rightarrow \mathrm{WW}^{*}$ and $\mathrm{H} \rightarrow \tau^{+} \tau^{-}$decay channels [6568]. Figure 11 shows the reconstructed di-photon invariant mass distribution of the $\mathrm{qq} \rightarrow \mathrm{qqH}, \mathrm{H} \rightarrow \gamma \gamma$ signal and the irreducible $\gamma \gamma \mathrm{jj}$ background with $\mathrm{m}_{\mathrm{H}}=115 \mathrm{GeV} / c^{2}$ for $60 \mathrm{fb}^{-1}$. The reducible backgrounds have not been evaluated yet for this channel. As for the associated $\mathrm{H} \rightarrow \gamma \gamma$ channels, a two photon invariant mass window of $3 \mathrm{GeV} / c^{2}$ was taken. The two-lepton-plus- $\mathrm{E}_{\mathrm{T}}^{\mathrm{miss}}$ final states from the $\mathrm{H} \rightarrow \mathrm{WW}^{*} \rightarrow \ell \nu_{\ell} \ell \nu_{\ell}$ channel were studied with full simulation for the signal and for the $t \bar{t}$ plus-jet background for $\mathrm{m}_{\mathrm{H}}=120 \mathrm{GeV} / c^{2}$, for which the $\mathrm{H} \rightarrow \mathrm{WW}^{*}$ branching fraction is small [66]. The WW spin correlations were used to suppress the backgrounds. For the $\mathrm{H} \rightarrow \tau^{+} \tau^{-}$decay modes the lepton-plus- $\tau$-jet final states were studied [67]. For this channel the mass resolution is crucial due to dominant QCD and electroweak Zjj backgrounds with $\mathrm{Z} \rightarrow \tau^{+} \tau^{-}$decays. Figure 12 shows the invariant mass distribution reconstructed from the $\tau$ jet, lepton and $\mathrm{E}_{\mathrm{T}}^{\mathrm{miss}}$ in lepton-plus- $\tau$-jet final state of the 


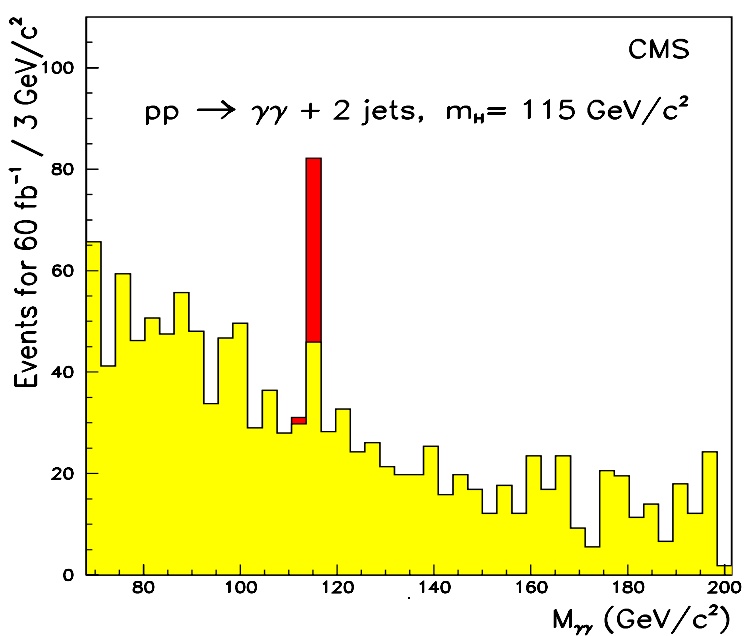

Fig. 11. Reconstructed di-photon invariant mass distribution of the qq $\rightarrow \mathrm{qqH}, \mathrm{H} \rightarrow \gamma \gamma$ signal (dark) and the background (light) with $\mathrm{m}_{\mathrm{H}}=115 \mathrm{GeV} / c^{2}$ for $60 \mathrm{fb}^{-1}$

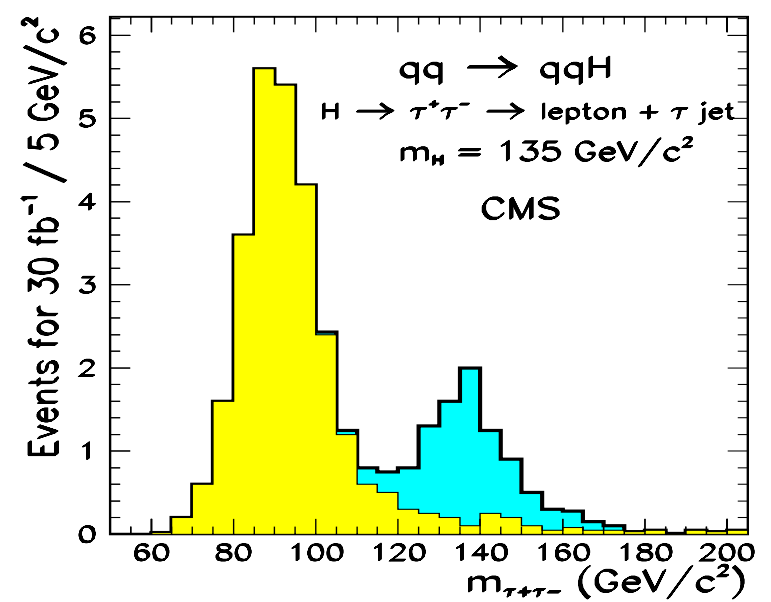

Fig. 12. Reconstructed $\tau \tau$ invariant mass distribution in the lepton-plus- $\tau$ jet final state in the qq $\rightarrow \mathrm{qqH}, \mathrm{H} \rightarrow \tau^{+} \tau^{-}$ signal (dark) and in the background (light) with $\mathrm{m}_{\mathrm{H}}=$ $135 \mathrm{GeV} / c^{2}$ for $30 \mathrm{fb}^{-1}$

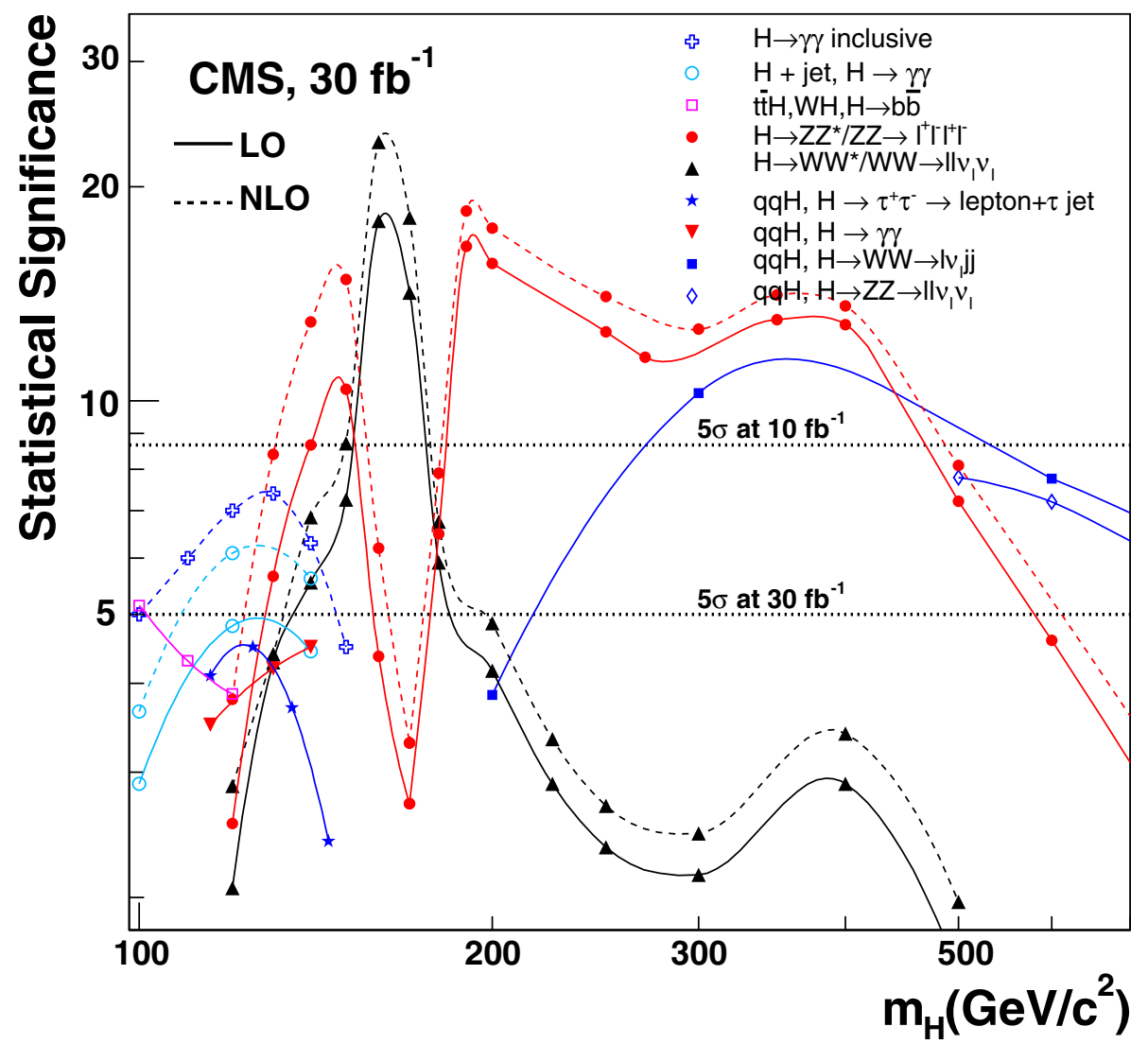

Fig. 13. Expected statistical significance with $30 \mathrm{fb}^{-1}$ for the SM Higgs boson as a function of $\mathrm{m}_{\mathrm{H}}$. The dashed lines show the result of the $\mathrm{NLO}$ analysis with $\mathrm{k}$ factors for signal and backgrounds. Systematic uncertainty of $5 \%$ due to background determination is included only for the $\mathrm{H} \rightarrow \mathrm{WW}^{*} / \mathrm{WW} \rightarrow \ell \ell \nu_{\ell} \nu_{\ell}$ channel. Poisson statistics was used to calculate the statistical significance for $\mathrm{H} \rightarrow \mathrm{ZZ}^{*} / \mathrm{ZZ} \rightarrow \ell^{+} \ell^{-} \ell^{\prime+} \ell^{\prime-}, \mathrm{H} \rightarrow \gamma \gamma$ in the WH production and for $\mathrm{H} \rightarrow \gamma \gamma$ and $\mathrm{H} \rightarrow \tau^{+} \tau^{-}$in the $\mathrm{qq} \rightarrow \mathrm{qqH}$ production

$\mathrm{H} \rightarrow \tau^{+} \tau^{-}$signal and the background for $\mathrm{m}_{\mathrm{H}}=135$ $\mathrm{GeV} / c^{2}$ with $30 \mathrm{fb}^{-1}$. For this point the mass resolution was found to be $11.3 \mathrm{GeV} / c^{2}$. The $\mathrm{W}+3$ jet background was suppressed with the $\tau$-jet identification criteria.

For Higgs boson masses larger than $500 \mathrm{GeV} / c^{2}$ the $\mathrm{H} \rightarrow \mathrm{WW} / \mathrm{ZZ}$ decay modes were used in the gauge boson fusion production process $[69,70]$. The $\ell \nu_{\ell} \mathrm{jj}$ and $\ell \ell \nu_{\ell} \nu_{\ell}$ final states were found to yield the highest sensitivity. The discovery potential can be extended down to $\mathrm{m}_{\mathrm{H}} \sim 300 \mathrm{GeV} / c^{2}$ with the $\mathrm{H} \rightarrow \mathrm{WW} \rightarrow \ell \nu_{\ell} \mathrm{jj}$ channel.

The statistical significance for the SM Higgs boson in all individual channels for $30 \mathrm{fb}^{-1}$ is shown in Fig. 13 for $100 \mathrm{GeV} / c^{2} \leq \mathrm{m}_{\mathrm{H}} \leq 800 \mathrm{GeV} / c^{2}$ and in Fig. 14 for $\mathrm{m}_{\mathrm{H}} \leq 150 \mathrm{GeV} / \mathrm{c}^{2}$. The results from the NLO analysis 


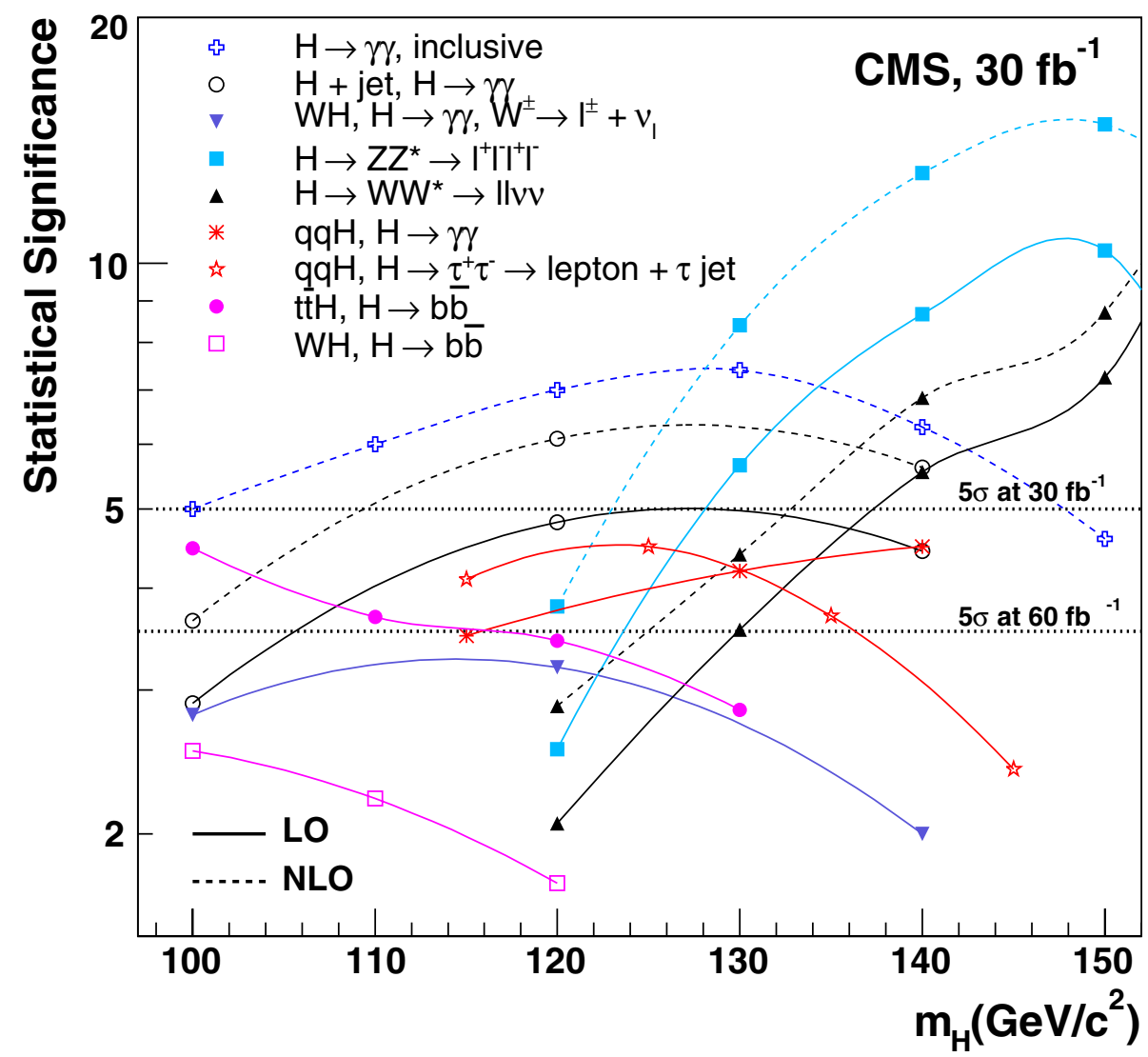

Fig. 14. The same as in Fig. 13 but for $\mathrm{m}_{\mathrm{H}}<150 \mathrm{GeV} / c^{2}$

with $\mathrm{k}$ factors for the signal and for the backgrounds are shown for the $\mathrm{H} \rightarrow \gamma \gamma$ channel in the inclusive and $\mathrm{H}+$ jet production and for the $\mathrm{H} \rightarrow \mathrm{ZZ}^{*} / \mathrm{ZZ} \rightarrow \ell^{+} \ell^{-} \ell^{\prime+} \ell^{\prime-}$ and $\mathrm{H} \rightarrow \mathrm{WW}^{*} / \mathrm{WW} \rightarrow \ell \ell \nu_{\ell} \nu_{\ell}$ channels. A systematic uncertainty of $5 \%$ on the background determination is included for the $\mathrm{H} \rightarrow \mathrm{WW}^{*} / \mathrm{WW} \rightarrow \ell \ell \nu_{\ell} \nu_{\ell}$ channel.

\subsection{Searches for heavy neutral MSSM Higgs bosons}

The $\mathrm{H}, \mathrm{A} \rightarrow \mu^{+} \mu^{-}$decay channel in the $\mathrm{gg} \rightarrow \mathrm{b} \overline{\mathrm{b}} \mathrm{H} / \mathrm{A}$ production was studied in [71] with full simulation and complete reconstruction. Figure 15 shows the reconstructed $\mu^{+} \mu^{-}$invariant mass distribution of the $\mathrm{pp} \rightarrow \mathrm{b} \overline{\mathrm{b}} \mathrm{H} / \mathrm{A}+\mathrm{X}$, $\mathrm{H} / \mathrm{A} \rightarrow \mu^{+} \mu^{-}$signal (dark), the $\mathrm{Z}, \gamma^{*}$ background (light) and the $\mathrm{t} \overline{\mathrm{t}}$ background (black) with $\mathrm{m}_{\mathrm{A}}=130 \mathrm{GeV} / c^{2}$, $\tan \beta=30$ for $20 \mathrm{fb}^{-1}$. The signal is a superposition of the $\mathrm{A}$ and $\mathrm{H}$ decays. The two-muon mass resolution of $1 \%$ is not good enough to resolve the $2 \mathrm{GeV} / c^{2}$ difference between the $\mathrm{A}$ and $\mathrm{H}$ boson masses in this parameter space point. Recent calculations show however that this mass difference could be as large as $5.5 \mathrm{GeV} / c^{2}$ for $\tan \beta \sim 30$ [72]. The dominant $\mathrm{Z}, \gamma^{*} \rightarrow \mu^{+} \mu^{-}$background was suppressed with b tagging. In the gg $\rightarrow \mathrm{b} \bar{b} \mathrm{H} / \mathrm{A}$ production process $\mathrm{b}$ jets are soft $\left(\mathrm{E}_{\mathrm{T}} \lesssim 50 \mathrm{GeV}\right)$ and distributed over a wide rapidity range. An algorithm combining the secondary vertex and impact parameter tagging methods without a requirement of a reconstructed calorimeter jet was used to obtain a better signal efficiency $(\sim 40 \%)$ than that with a b-jet-tagging method. Because this b tagging technique does not require a jet to be reconstructed in the calorimeter the $t \bar{t}$ background was suppressed efficiently vetoing central jets.

The e $\mu, \ell^{+} \ell^{-}[48]$, lepton-plus- $\tau$-jet [47] and two- $\tau$ jet final states [15] were studied for the $\mathrm{H}, \mathrm{A} \rightarrow \tau^{+} \tau^{-}$ decay modes. Figure 16 shows the reconstructed $\tau^{+} \tau^{-}$ invariant mass distribution in the $\mathrm{e} \mu$ final state in the $\mathrm{pp} \rightarrow \mathrm{b} \overline{\mathrm{b}} \mathrm{H} / \mathrm{A}+\mathrm{X}, \mathrm{H} / \mathrm{A} \rightarrow \tau^{+} \tau^{-}$signal (dark) and in the background (light) with $\mathrm{m}_{\mathrm{A}}=200 \mathrm{GeV} / c^{2}, \tan \beta=20$ for $30 \mathrm{fb}^{-1}$. A similar distribution is shown in Fig. 17 for the two- $\tau$-jet final state in the $\mathrm{pp} \rightarrow \mathrm{b} \overline{\mathrm{b}} \mathrm{H} / \mathrm{A}+\mathrm{X}$, $\mathrm{H} / \mathrm{A} \rightarrow \tau^{+} \tau^{-}$signal (dark), in the total background (light) and in the QCD multi-jet background (dashed) with $\mathrm{m}_{\mathrm{A}}=500 \mathrm{GeV} / c^{2}, \tan \beta=30$ for $60 \mathrm{fb}^{-1}$. The identification of the hadronic $\tau$ decays for the two- $\tau$-jet final state was performed with full simulation including the Level-1 trigger and HLT simulations [15]. For the suppression of the $\mathrm{Z}, \gamma^{*} \rightarrow \tau^{+} \tau^{-}$background and for further suppression the QCD multi-jet background, one associated b jet was reconstructed and tagged with the impact parameter method. For a background rejection factor of 100, the overall b-jet tagging efficiencies were found to be $\sim 20 \%$ due to the small $\mathrm{E}_{\mathrm{T}}$ scale of the associated $\mathrm{b}$ jets and due to the importance of the forward tracking regions in the impact parameter measurements. The $\tau$ tagging with impact parameter measurement was also used to suppress the $\mathrm{Z} \rightarrow \ell^{+} \ell^{-}$background in the two-lepton final state and to further suppress the QCD multi-jet background in the two- $\tau$-jet final state. The $t \bar{t}$ and $\mathrm{Wt}$ backgrounds 


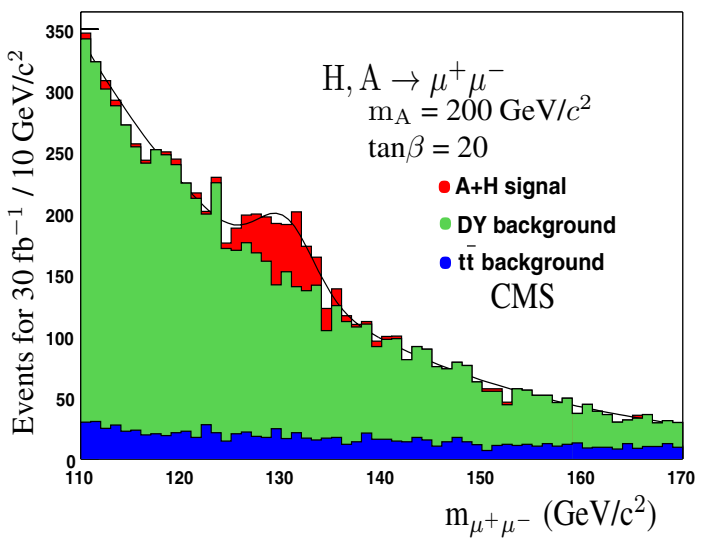

Fig. 15. Reconstructed di-muon invariant mass distribution of the $\mathrm{pp} \rightarrow \mathrm{b} \overline{\mathrm{b}} \mathrm{H} / \mathrm{A}+\mathrm{X}, \mathrm{H} / \mathrm{A} \rightarrow \mu^{+} \mu^{-}$signal (dark), the $\mathrm{Z}, \gamma^{*}$ background (light) and the $\mathrm{t} \overline{\mathrm{t}}$ background (black) with $\mathrm{m}_{\mathrm{H}}=130 \mathrm{GeV} / c^{2}, \tan \beta=30$ for $20 \mathrm{fb}^{-1}$

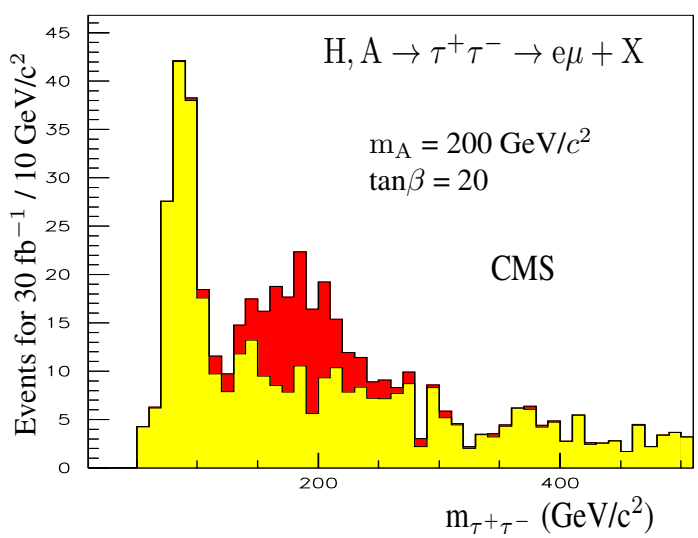

Fig. 16. Reconstructed $\tau \tau$ invariant mass distribution in the $\mathrm{e} \mu$ final state in the $\mathrm{pp} \rightarrow \mathrm{b} \overline{\mathrm{b}} \mathrm{H} / \mathrm{A}+\mathrm{X}, \mathrm{H} / \mathrm{A} \rightarrow \tau^{+} \tau^{-}$signal (dark) and in the background (light) with $\mathrm{m}_{\mathrm{A}}=200 \mathrm{GeV} / c^{2}$ and $\tan \beta=20$ for $30 \mathrm{fb}^{-1}$

with genuine b's and $\tau$ 's from the $\mathrm{W} \rightarrow \tau \nu_{\tau}$ decays were suppressed with a veto on a second central jet.

The uncertainty of the $\tan \beta$ measurement from the event rates in the $\mathrm{H}, \mathrm{A} \rightarrow \tau^{+} \tau^{-}$channels was found to be $17.2 \%$ for the two- $\tau$-jet final state, $10.8 \%$ for the leptonplus- $\tau$-jet final state and $12.7 \%$ for the two-lepton final state for $\mathrm{m}_{\mathrm{A}}=200 \mathrm{GeV} / c^{2}, \tan \beta=20$ with $30 \mathrm{fb}^{-1}$ assuming a $20 \%$ theoretical uncertainty due the choice of the scale [7] and a $5 \%$ uncertainty in the luminosity measurement.

\subsection{Searches for charged Higgs bosons}

Searches for the charged Higgs bosons in têt events $\left(\mathrm{m}_{\mathrm{H}^{ \pm}}<\right.$ $\mathrm{m}_{\text {top }}$ ) were studied in [73]. A lepton from one of the top quarks and hadronic $\tau$ decays from the $\mathrm{H}^{ \pm} \rightarrow \tau \nu_{\tau}$ decay channel are required. As the Higgs boson mass cannot be reconstructed in this channel the signal was obtained as an excess of $\tau$ 's in the t $\bar{t}$ events relative to electrons and muons.

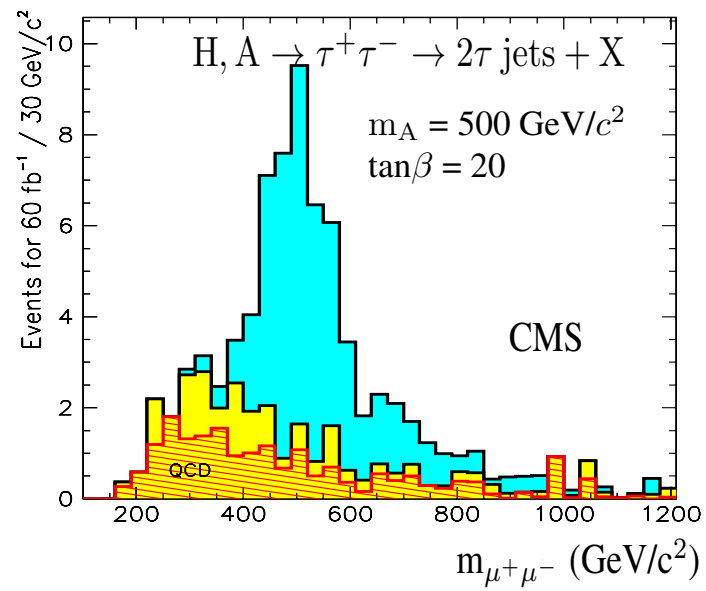

Fig. 17. Reconstructed $\tau^{+} \tau^{-}$invariant mass distribution in the two- $\tau$-jet final state in the $\mathrm{pp} \rightarrow \mathrm{b} \overline{\mathrm{b}} \mathrm{H} / \mathrm{A}+\mathrm{X}, \mathrm{H} / \mathrm{A} \rightarrow \tau^{+} \tau^{-}$ signal (dark), the total background (light) and in the QCD multi-jet background (dashed) with $\mathrm{m}_{\mathrm{A}}=500 \mathrm{GeV} / c^{2}$ and $\tan \beta=30$ for $60 \mathrm{fb}^{-1}$

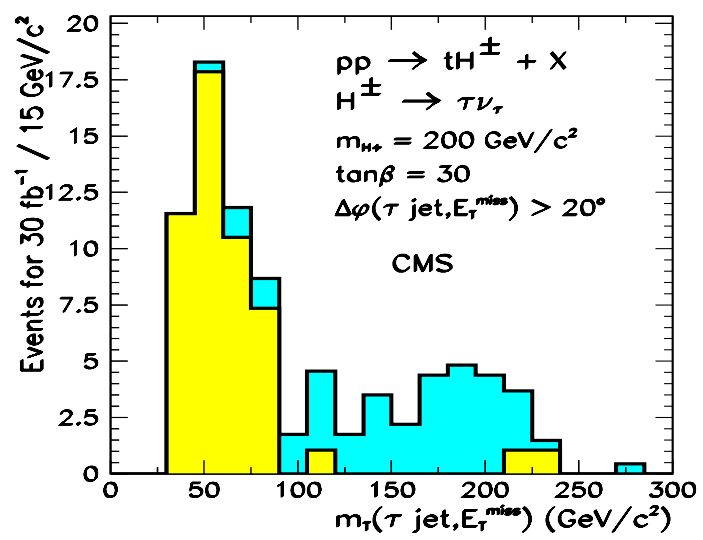

Fig. 18. Transverse mass reconstructed from the $\tau$ jet and $\mathrm{E}_{\mathrm{T}}^{\text {miss }}$ in the $\mathrm{gg} \rightarrow \mathrm{tH}^{ \pm}, \mathrm{H}^{ \pm} \rightarrow \tau \nu_{\tau}$ signal (dark) and in the background (light) with $\mathrm{m}_{\mathrm{H}^{ \pm}}=200 \mathrm{GeV} / c^{2}$ and $\tan \beta=30$ for $30 \mathrm{fb}^{-1}$

For the heavier charged Higgs bosons $\left(\mathrm{m}_{\mathrm{H}^{ \pm}}>\mathrm{m}_{\mathrm{top}}\right)$, the $\mathrm{H}^{ \pm} \rightarrow \tau \nu_{\tau}$ and $\mathrm{H}^{ \pm} \rightarrow$ tb decay channels were studied in associated production $\mathrm{gb} \rightarrow \mathrm{tH}^{ \pm}$. To suppress the $\mathrm{t} \overline{\mathrm{t}}$ and Wt backgrounds with genuine $\tau$ 's (for the $\mathrm{H}^{ \pm} \rightarrow \tau \nu_{\tau}$ decay channels with hadronic $\tau$ decays), the helicity correlations were exploited requiring at least $80 \%$ of the visible $\tau$-jet energy to be carried by a single charged pion. Figure 18 shows the transverse mass reconstructed from the $\tau$ jet and $\mathrm{E}_{\mathrm{T}}^{\mathrm{miss}}$ in the $\mathrm{gg} \rightarrow \mathrm{tH}^{ \pm}, \mathrm{H}^{ \pm} \rightarrow \tau \nu_{\tau}, \tau \rightarrow$ hadrons $+\nu_{\tau}$ signal (dark) and in the background (light) with $\mathrm{m}_{\mathrm{H}^{ \pm}}=$ $200 \mathrm{GeV} / c^{2}$ and $\tan \beta=30$ for $30 \mathrm{fb}^{-1}$ [74]. In these purely hadronic events the $\mathrm{E}_{\mathrm{T}}^{\text {miss }}$ originates mainly from the $\mathrm{H}^{ \pm} \rightarrow \tau \nu_{\tau}$ decay (for large $\mathrm{E}_{\mathrm{T}}^{\tau}$ jet cut) yielding an endpoint in the transverse mass at $\mathrm{m}_{\mathrm{H}^{ \pm}}$for the signal and at $\mathrm{m}_{\mathrm{W}}$ for the residual backgrounds. These backgrounds can be further suppressed with a cut on the relative azimuthal angle $\Delta \varphi$ between the $\tau$ jet and the $\mathrm{E}_{\mathrm{T}}^{\text {miss }}$ direction $\left(\Delta \varphi>20^{\circ}\right.$ in Fig. 18). The dominating $\mathrm{H}^{ \pm} \rightarrow$ tb decay channel in the $\mathrm{gb} \rightarrow \mathrm{tH}^{ \pm}$production was studied in 


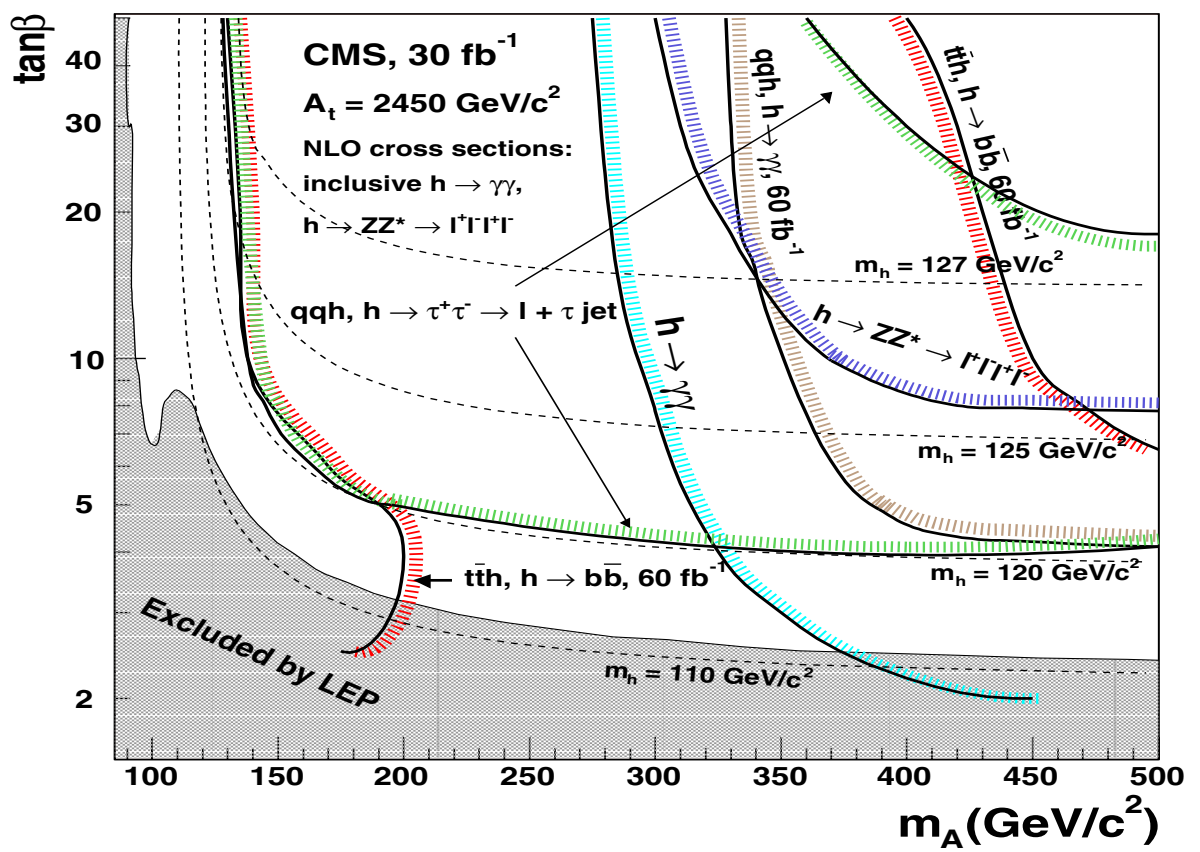

Fig. 19. The $5 \sigma$-discovery potential for the lighter scalar MSSM Higgs boson as a function of $\mathrm{m}_{\mathrm{A}}$ and $\tan \beta$ for $30 \mathrm{fb}^{-1}$ with $\mathrm{A}_{\mathrm{t}}=2450 \mathrm{GeV} / \mathrm{c}^{2}$. The

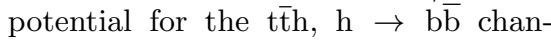
nel and for the $\mathrm{h} \rightarrow \gamma \gamma$ channel in the $\mathrm{qq} \rightarrow \mathrm{qqh}$ process are shown for $60 \mathrm{fb}^{-1}$. Poisson statistics were used to calculate the statistical significance for the $\mathrm{h} \rightarrow \mathrm{ZZ}^{*} \rightarrow \ell^{+} \ell^{-} \ell^{\prime+} \ell^{\prime-}$ channel and for the $\mathrm{h} \rightarrow \gamma \gamma$ and $\mathrm{h} \rightarrow \tau^{+} \tau^{-}$decay channels in the qq $\rightarrow$ qqh production. Systematic uncertainties are not included

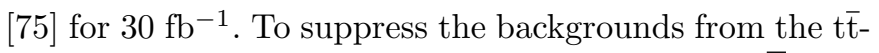
plus-jet production (containing the irreducible t $t \bar{t} b \bar{b}$ component) one leptonic and one hadronic top quark was reconstructed. The Higgs boson mass is given by one of the top quarks and one b-tagged jet leading to a large combinatorial background. Due to a modest Higgs boson mass resolution in this channel the systematic uncertainties in the background determination (not included in [75]) reduce the discovery region to large $\tan \beta$ values $(\gtrsim 50)$. Heavy charged Higgs bosons are accessible at large $\tan \beta$ also in the s-channel production $\mathrm{q} \overline{\mathrm{q}} \rightarrow \mathrm{H}^{ \pm} \rightarrow \tau \nu_{\tau}$ with hadronic $\tau$ decays as was shown in [8]. The helicity correlations and transverse mass reconstruction were used to suppress the large $\mathrm{q} \overline{\mathrm{q}} \rightarrow \mathrm{W} \rightarrow \tau \nu_{\tau}$ background.

The statistical uncertainty of the Higgs boson mass measurement from the transverse mass distributions for $30 \mathrm{fb}^{-1}$ was found to be $\lesssim 2 \%$ in the $\mathrm{gb} \rightarrow \mathrm{tH}^{ \pm}$, $\mathrm{H}^{ \pm} \rightarrow \tau \nu_{\tau}$ channel with $\tan \beta>30$ and $\mathrm{m}_{\mathrm{H}^{ \pm}}=400$ $\mathrm{GeV} / c^{2}$ and $\lesssim 15 \%$ in the $\mathrm{q} \overline{\mathrm{q}} \rightarrow \mathrm{H}^{ \pm} \rightarrow \tau \nu_{\tau}$ channel with $\tan \beta>20$ and $\mathrm{m}_{\mathrm{H}^{ \pm}}=200 \mathrm{GeV} / c^{2}$. The uncertainty of the $\tan \beta$ measurement from the event rates in the $\mathrm{gb} \rightarrow \mathrm{tH}^{ \pm}, \mathrm{H}^{ \pm} \rightarrow \tau \nu_{\tau}$ process for $30 \mathrm{fb}^{-1}$ was found to be $14 \%$ with $\tan \beta=30$ and $\mathrm{m}_{\mathrm{H}^{ \pm}}=200 \mathrm{GeV} / c^{2}$ and $20 \%$ with $\mathrm{m}_{\mathrm{H}^{ \pm}}=400 \mathrm{GeV} / c^{2}$ assuming a $20 \%$ theoretical uncertainty [7] and a $5 \%$ uncertainty in the luminosity measurement. In the $\mathrm{q} \overline{\mathrm{q}} \rightarrow \mathrm{H}^{ \pm} \rightarrow \tau \nu_{\tau}$ channel the $\tan \beta$ uncertainty was found to be $\lesssim 15 \%$ with $\tan \beta>20$ and $\mathrm{m}_{\mathrm{H}^{ \pm}}=200 \mathrm{GeV} / c^{2}$ for $30 \mathrm{fb}^{-1}[8]$.

\subsection{Discovery potential in the MSSM}

Figures 19 and 20 show the discovery potential for the lighter scalar MSSM Higgs boson $h$ as a function of $\mathrm{m}_{\mathrm{A}}$ and $\tan \beta$, assuming $\mathrm{A}_{\mathrm{t}}=2450 \mathrm{GeV} / c^{2}, \mathrm{~m}_{\mathrm{top}}=$ $175 \mathrm{GeV} / c^{2}$ and $\mathrm{m}_{\mathrm{SUSY}}=1 \mathrm{TeV} / c^{2}$ for 30 and $100 \mathrm{fb}^{-1}$, respectively. The decay channels included in these figures are the inclusive $\mathrm{h} \rightarrow \gamma \gamma, \mathrm{h} \rightarrow \mathrm{ZZ}^{*} \rightarrow \ell^{+} \ell^{-} \ell^{\prime+} \ell^{\prime-}, \mathrm{h} \rightarrow \gamma \gamma$ in the associated production $\mathrm{Wh}, \mathrm{h} \rightarrow \mathrm{b} \overline{\mathrm{b}}$ in the asso-

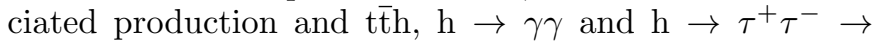
$\ell+\tau$ jet in the gauge boson fusion qq $\rightarrow$ qqh. The NLO cross sections were used for the inclusive $h \rightarrow \gamma \gamma$ and

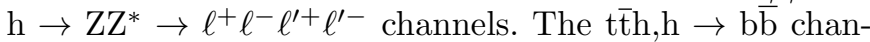
nel covers the region $\mathrm{m}_{\mathrm{A}} \gtrsim 150 \mathrm{GeV} / c^{2}$ and $\tan \beta \gtrsim 10$ for $60 \mathrm{fb}^{-1}$. A region at large $\mathrm{m}_{\mathrm{A}}$ and $\tan \beta$ is not accessible with this channel due to the decreasing $\mathrm{h} \rightarrow \mathrm{b} \overline{\mathrm{b}}$ branching fraction with increasing $m_{h}$. For the same reason the sensitivity in this channel is larger stop mixing is small. As the branching fraction for the $\mathrm{h} \rightarrow \mathrm{ZZ}^{*}$ decay decreases fast with decreasing $m_{h}$, the discovery potential for the $\mathrm{h} \rightarrow \mathrm{ZZ}^{*} \rightarrow \ell^{+} \ell^{-} \ell^{\prime+} \ell^{\prime-}$ channel is particularly sensitive to the MSSM parameters (mainly to the amount of stop mixing) through the maximum value of $\mathrm{m}_{\mathrm{h}}\left(\sim 127 \mathrm{GeV} / c^{2}\right.$ for maximal stop mixing). For the MSSM scenarios where $\mathrm{m}_{\mathrm{h}}^{\max } \lesssim 120 \mathrm{GeV} / c^{2}$ no sensitivity is expected in this channel. For the $\mathrm{h} \rightarrow \tau^{+} \tau^{-}$decay channel in the gauge boson fusion the region $\tan \beta \gtrsim 4$ is accessible for $\mathrm{m}_{\mathrm{A}} \gtrsim 150 \mathrm{GeV} / \mathrm{c}^{2}$ apart from a small region with $\tan \beta \gtrsim 20, \mathrm{~m}_{\mathrm{A}} \gtrsim 400 \mathrm{GeV} / c^{2}$ which requires higher integrated luminosity. For $60 \mathrm{fb}^{-1}$, the $\mathrm{h} \rightarrow \gamma \gamma$ decay channel in the qq $\rightarrow$ qqh production covers the region $\mathrm{m}_{\mathrm{A}} \gtrsim 400 \mathrm{GeV} / c^{2}$. The parameter space for $90 \lesssim \mathrm{m}_{\mathrm{A}} \lesssim 130 \mathrm{GeV} / c^{2}$, where the lighter scalar is no more SM-like, is out of reach of the channels discussed in this Section. Investigations are in progress to cover this region with the $\mathrm{h} \rightarrow \mu^{+} \mu^{-}$and $\mathrm{h} \rightarrow \tau^{+} \tau^{-}$decay channels in the associated production gg $\rightarrow \mathrm{b} \bar{b} h$, taking advantage of the b- and $\tau$-tagging methods and mass reconstruction from $\tau$ 's.

Figure 21 shows the $5 \sigma$-discovery potential for the heavy neutral MSSM Higgs bosons $\mathrm{H}$ and $\mathrm{A}$ in the $\mathrm{H}, \mathrm{A} \rightarrow$ $\tau^{+} \tau^{-}$and $\mathrm{H}, \mathrm{A} \rightarrow \mu^{+} \mu^{-}$decay channels as a function of 

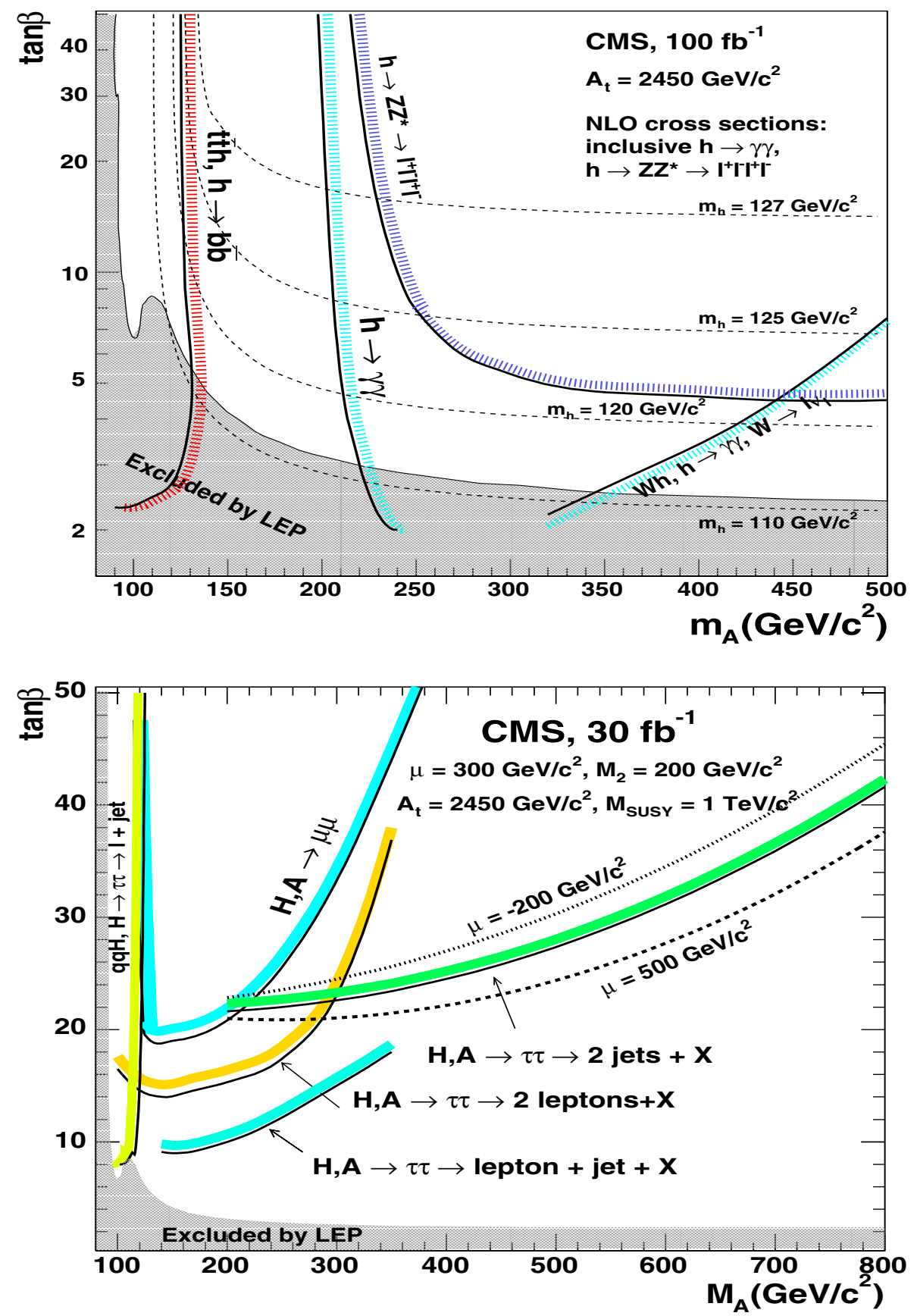

Fig. 20. The $5 \sigma$-discovery potential for the lighter scalar MSSM Higgs boson as a function of $\mathrm{m}_{\mathrm{A}}$ and $\tan \beta$ for $100 \mathrm{fb}^{-1}$ with $\mathrm{A}_{\mathrm{t}}=2450 \mathrm{GeV} / \mathrm{c}^{2}$. Poisson statistics were used to calculate the statistical significance for the $\mathrm{h} \rightarrow \mathrm{ZZ}^{*} \rightarrow \ell^{+} \ell^{-} \ell^{\prime+} \ell^{\prime-}$ channel and for the $\mathrm{h} \rightarrow \gamma \gamma$ decay channel in the Wh production. Systematic uncertainties are not included

Fig. 21. The $5 \sigma$-discovery potential for the heavy neutral MSSM Higgs bosons $\mathrm{H}$ and $\mathrm{A}$ with $\mathrm{A}_{\mathrm{t}}=2450 \mathrm{GeV} / c^{2}$ for $30 \mathrm{fb}^{-1}$. Poisson statistics were used to calculate the statistical significance for the $\mathrm{H} \rightarrow \tau^{+} \tau^{-}$channels in the $\mathrm{qq} \rightarrow \mathrm{qqH}$ production. Systematic uncertainties are not included
$\mathrm{m}_{\mathrm{A}}$ and $\tan \beta$. The discovery potential for the two-lepton and lepton-plus- $\tau$-jet final states from the $\mathrm{H}, \mathrm{A} \rightarrow \tau^{+} \tau^{-}$ decay channel are shown for $30 \mathrm{fb}^{-1}$ while those for the two- $\tau$-jet final states and for the $\mathrm{H}, \mathrm{A} \rightarrow \mu^{+} \mu^{-}$channel are shown for $60 \mathrm{fb}^{-1}$. For the two-lepton final states with $\mathrm{m}_{\mathrm{A}} \lesssim 200 \mathrm{GeV} / c^{2}$, the $\mathrm{e} \mu$ component yields a higher sensitivity than the $\ell \ell$ final state, which is overwhelmed with the direct $\mathrm{Z} \rightarrow \ell^{+} \ell^{-}$background near the $\mathrm{Z}$ peak, reducible only with $\tau$ tagging. Therefore the $\mathrm{e}^{+} \mathrm{e}^{-}$and $\mu^{+} \mu^{-}$final states were ignored for $\mathrm{m}_{\mathrm{A}} \lesssim 200 \mathrm{GeV} / c^{2}$. The effect of the variation of the $\mu$ parameter between 200 and $+500 \mathrm{GeV} / c^{2}$ on the discovery potential for the $\mathrm{H} \rightarrow \tau^{+} \tau^{-} \rightarrow$ two $\tau$-jet channel is also shown in Fig. 21 .
Figure 22 shows the $5 \sigma$-discovery potential for the charged Higgs bosons as a function of $\mathrm{m}_{\mathrm{A}}$ and $\tan \beta$ for the $\mathrm{H}^{ \pm} \rightarrow \tau \nu_{\tau}$ decay channel with hadronic $\tau$ decays in the associated production $\mathrm{gb} \rightarrow \mathrm{tH}^{ \pm}$, in the $\mathrm{t} \overline{\mathrm{t}}$ events and in the direct production $\mathrm{q} \overline{\mathrm{q}} \rightarrow \mathrm{H}^{ \pm}$and for the $\mathrm{H}^{ \pm} \rightarrow \mathrm{tb}$ decay channel in the associated production $\mathrm{gb} \rightarrow \mathrm{tH}^{ \pm}$. The potential is shown with $\mathrm{A}_{\mathrm{t}}=2450 \mathrm{GeV} / c^{2}$ and for an integrated luminosity of $30 \mathrm{fb}^{-1}$. The region around the top quark mass $\left(140 \mathrm{GeV} / c^{2} \lesssim \mathrm{m}_{\mathrm{A}} \lesssim 200 \mathrm{GeV} / c^{2}\right)$ is still under investigation. The $\mathrm{gg} \rightarrow \mathrm{tbH}^{ \pm}$production process can be used with the $\mathrm{H}^{ \pm} \rightarrow \tau \nu_{\tau}$ decay mode. 


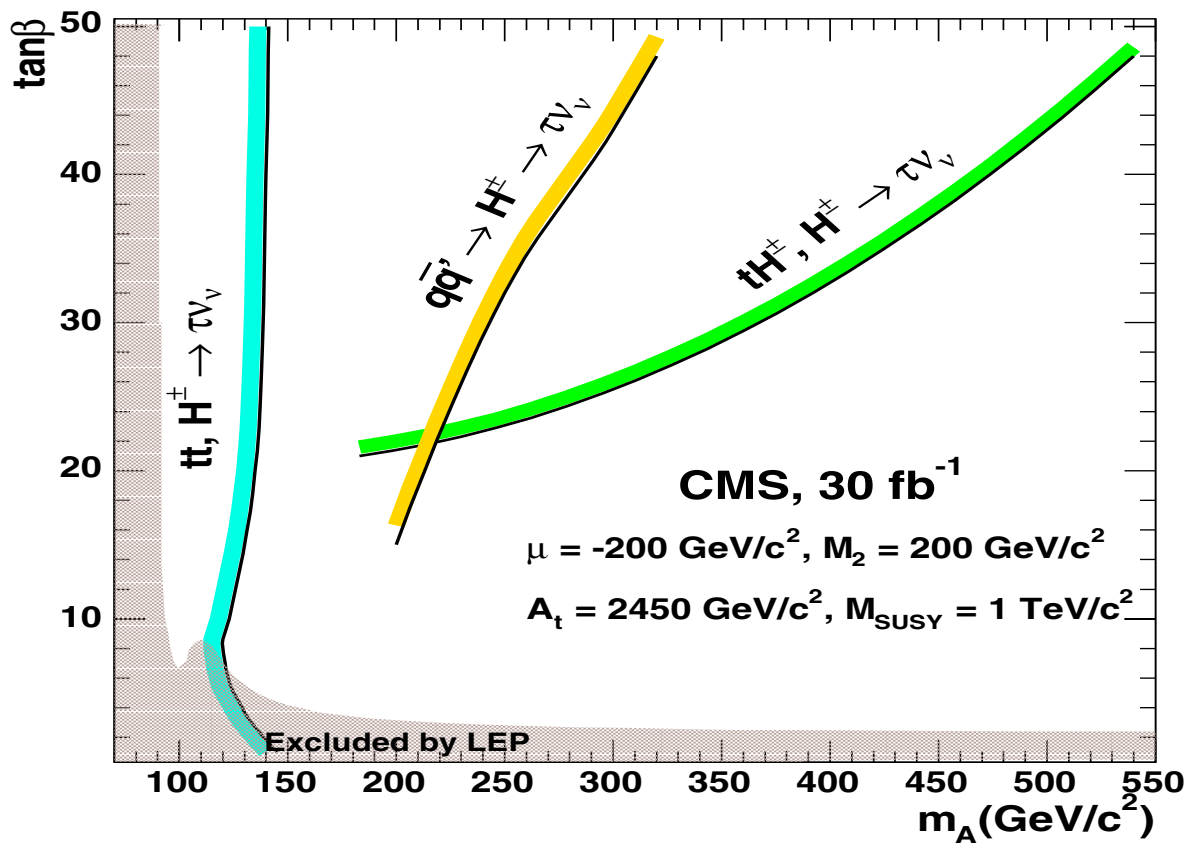

Fig. 22. The $5 \sigma$-discovery potential for the charged Higgs bosons in the gb $\rightarrow$ $\mathrm{tH}^{ \pm}$and $\mathrm{t} \overline{\mathrm{t}}$ production processes as a function of $\mathrm{m}_{\mathrm{A}}$ and $\tan \beta$ with $30 \mathrm{fb}^{-1}$. Poisson statistics were used to calculate the statistical significance for the $\mathrm{H}^{ \pm} \rightarrow \tau \nu_{\tau}$ decay channel in the gb $\rightarrow$ $\mathrm{tH}^{ \pm}$production. Systematic uncertainties are not included

\section{Specific SUSY searches}

\subsection{Higgs boson decays into gauginos}

The four-lepton final state from the $\mathrm{H}, \mathrm{A} \rightarrow \chi_{2} \chi_{2}$ decay channel with $\chi_{2} \rightarrow \chi_{1} \ell^{+} \ell^{-}$was studied in [76]. Figure 23 shows the four-lepton invariant mass distribution of the $\mathrm{H}, \mathrm{A} \rightarrow \chi_{2} \chi_{2}$ signal and the background with $\mathrm{m}_{\mathrm{A}}=350 \mathrm{GeV} / c^{2}, \tan \beta=5, \mathrm{M}_{2}=120 \mathrm{GeV} / c^{2}, \mu=-$ $500 \mathrm{GeV} / c^{2}, \mathrm{M}_{\tilde{\mathrm{q}}, \tilde{\mathrm{g}}}=1000 \mathrm{GeV} / c^{2}$ and $\mathrm{M}_{\tilde{\ell}}=250 \mathrm{GeV} / c^{2}$ for $100 \mathrm{fb}^{-1}[76]$. Lepton isolation, $\mathrm{E}_{\mathrm{T}}^{\mathrm{miss}}$ lower cut and jet veto were used to suppress the SM ( $\mathrm{ZZ}, \mathrm{Zb} \overline{\mathrm{b}}, \mathrm{Zc} \overline{\mathrm{c}}, \mathrm{t} \overline{\mathrm{t}})$ and the sparticle $(\tilde{\mathrm{q}}, \tilde{\mathrm{g}})$ production backgrounds. Observation of the four-lepton signal in this channel requires understanding of origin, magnitude and shape of the various SM and SUSY backgrounds as the four-lepton invariant mass spectrum does not exhibit any kinematically obvi-

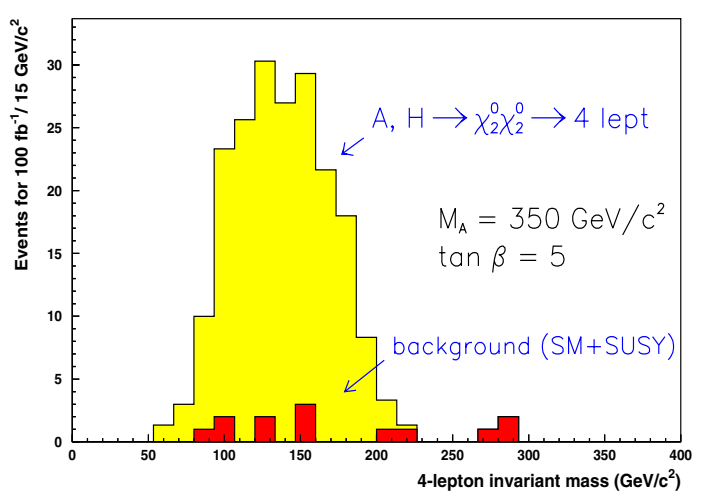

Fig. 23. Invariant mass distribution reconstructed from four leptons of the $\mathrm{H}, \mathrm{A} \rightarrow \chi_{2} \chi_{2} \rightarrow \ell^{+} \ell^{-} \ell^{\prime+} \ell^{\prime-}+\mathrm{X}$ signal (light) and the SM+SUSY background (dark) with $\mathrm{m}_{\mathrm{A}}=350 \mathrm{GeV} / c^{2}$, $\tan \beta=5$ for $100 \mathrm{fb}^{-1}$

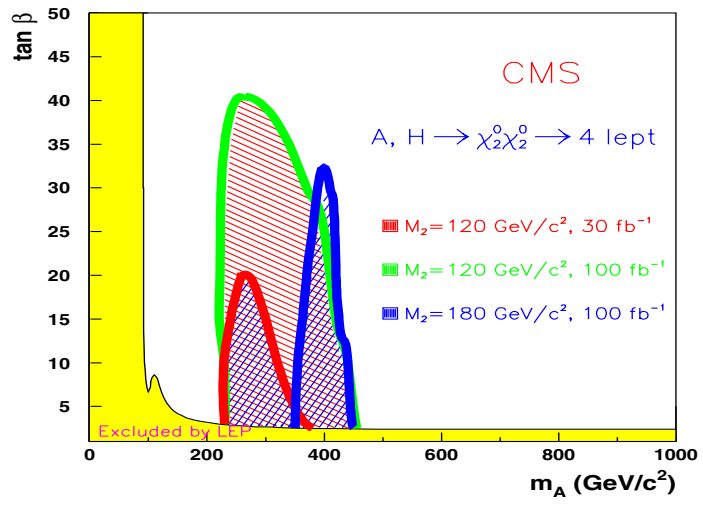

Fig. 24. The $5 \sigma$-discovery potential for the $\mathrm{H}, \mathrm{A} \rightarrow \chi_{2} \chi_{2} \rightarrow$ $\ell^{+} \ell^{-} \ell^{\prime+} \ell^{\prime-}+\mathrm{X}$ channel with $\mathrm{M}_{2}=120$ and $180 \mathrm{GeV} / c^{2}$ for 30 and $100 \mathrm{fb}^{-1}$. Systematic uncertainties are not included

ous features. Examples of the $5 \sigma$-discovery reach for this channel are shown in Fig. 24.

For the charged Higgs bosons, the $\mathrm{gb} \rightarrow \mathrm{tH}^{ \pm}, \mathrm{H}^{ \pm} \rightarrow$ $\chi_{2,3} \chi_{1,2}^{ \pm}$channel was studied in [77] in the three-lepton final state $\left(\chi_{2} \rightarrow \chi_{1} \ell^{+} \ell^{-}\right.$and $\left.\chi^{ \pm} \rightarrow \chi_{1} \ell^{ \pm} \nu_{\ell}\right)$. To sup-

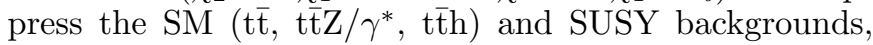
a hadronically decaying associated top quark was reconstructed. This channel was found to be accessible only for a limited fraction of the parameter space, namely for light sleptons and for $|\mu| \lesssim 150 \mathrm{GeV} / c^{2}$, i.e. near the LEP lower limit [77].

The invisible final states from Higgs boson decays in the qq $\rightarrow$ qqh production process were studied in [78]. Besides the backgrounds for which the $\mathrm{E}_{\mathrm{T}}^{\mathrm{miss}}$ originates from $\mathrm{W}$ and $\mathrm{Z}$ decays (electroweak or QCD production of $\mathrm{Zjj}$ and $\mathrm{Wjj}$ ) there is a large potential background from QCD multi-jet events with the $\mathrm{E}_{\mathrm{T}}^{\mathrm{miss}}$ originating from measurement fluctuations or from heavy flavour decays. This 


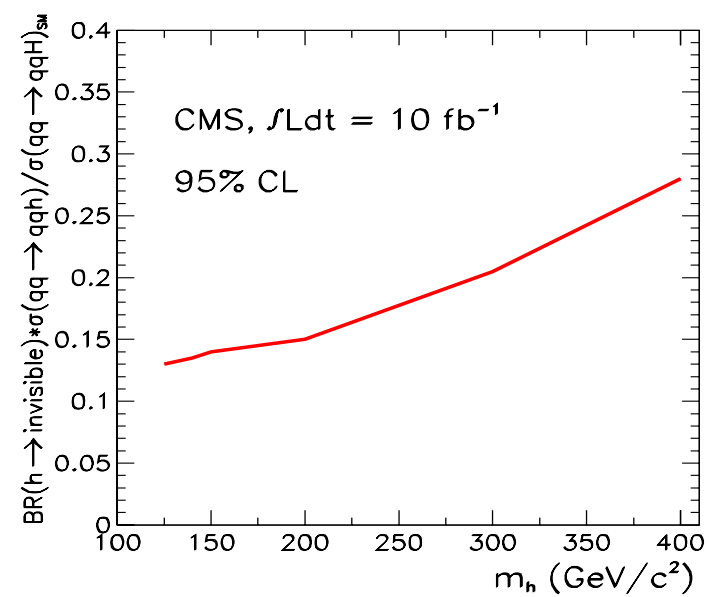

Fig. 25. Expected 95\% CL upper limit on the $\mathrm{h} \rightarrow$ invisible cross-section normalized on SM cross-section as a function of $\mathrm{m}_{\mathrm{H}}$ for $10 \mathrm{fb}^{-1}$

multi-jet background can be suppressed with an upper limit on the angle $\Delta \varphi$ between the two tagging jets. For the QCD multi-jet background, these jets tend to be in a back-to-back configuration, with $\mathrm{E}_{\mathrm{T}}^{\mathrm{miss}}$ following one of the jets, whilst for the qq $\rightarrow$ qqh production the tagging jets tend to recoil against the $\mathrm{E}_{\mathrm{T}}^{\mathrm{miss}}$ from the Higgs boson decay. The background levels can ultimately be obtained with a systematic uncertainty of $\sim 3 \%$ from the data themselves, taking advantage of $\mathrm{Wjj}$ and $\mathrm{Zjj}$ events with leptons reconstructed in $\mathrm{Z} \rightarrow \ell \ell$ and $\mathrm{W} \rightarrow \ell \nu_{\ell}$ decays [79]. The SUSY backgrounds, not included in this study,

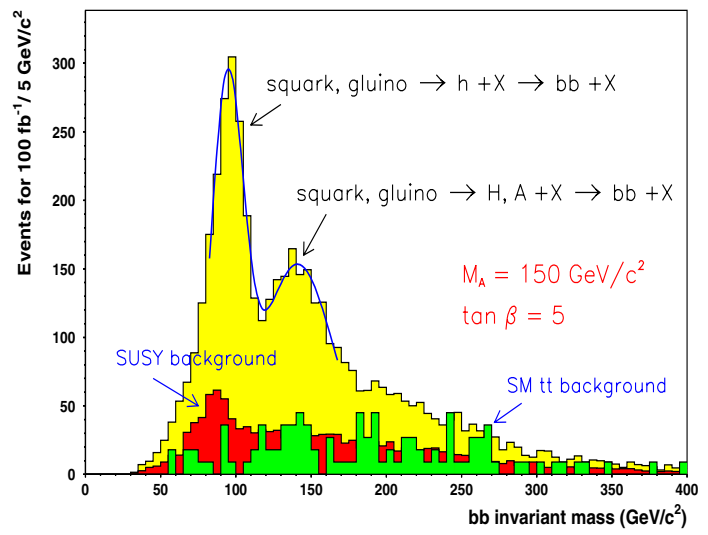

Fig. 26. Invariant mass reconstructed from two b jets in the $\chi_{2} \rightarrow \mathrm{h}, \mathrm{H}, \mathrm{A}+\chi_{1}, \mathrm{~h}, \mathrm{H}, \mathrm{A} \rightarrow \mathrm{b} \overline{\mathrm{b}}$ signal (light) and the SUSY (dark) and SM (gray) background with $\mathrm{m}_{\mathrm{A}}=150 \mathrm{GeV} / c^{2}$, $\tan \beta=5$ for $100 \mathrm{fb}^{-1}$

are expected to be suppressed with the central jet veto as in these backgrounds the $\mathrm{E}_{\mathrm{T}}^{\mathrm{miss}}$ is accompanied with at least two hadronic jets. Figure 25 shows the model independent $95 \%$ CL sensitivity to the $\mathrm{h} \rightarrow$ invisible signal for $10 \mathrm{fb}^{-1}$. The current combined limits on the $\chi_{1}^{0}$ and Higgs boson masses in a general SUSY model are such that, for non-universal gaugino masses at high scale, it is still kinematically possible for a relatively light Higgs boson to decay into a pair of $\chi_{1}^{0}$ with the branching fraction above $70 \%$, which still conforms with the relic density and $(\mathrm{g}-2)_{\mu}$ constraints [80].

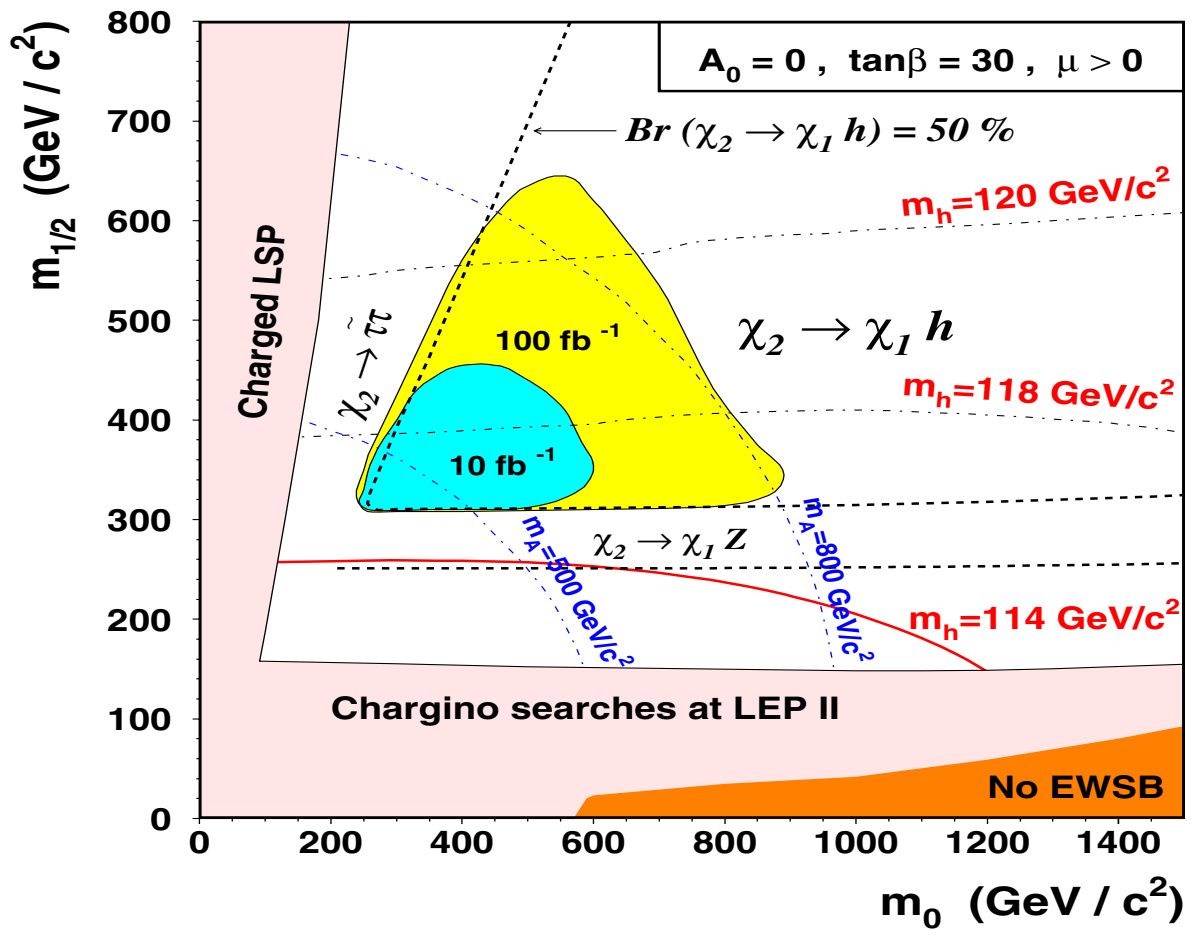

Fig. 27. The $5 \sigma$-discovery potential for $\mathrm{h} \rightarrow \mathrm{b} \overline{\mathrm{b}}$ from $\chi_{2} \rightarrow \mathrm{h} \chi_{1}$ in the squark and gluino cascades with $\mathrm{A}_{0}=0$, $\tan \beta=30$ and $\mu>0$ for 10 and $100 \mathrm{fb}^{-1}$. The regions excluded in theory and by LEP searches are also shown in the figure. Systematic uncertainties are not included 


\subsection{Higgs bosons from the decays of gauginos}

The production of $\mathrm{h}, \mathrm{H}, \mathrm{A}$ and $\mathrm{H}^{ \pm}$from gaugino decays in the MSSM was studied in [81]. Figure 26 shows the distribution of the reconstructed invariant $\mathrm{b} \overline{\mathrm{b}}$ mass of the $\chi_{2} \rightarrow \mathrm{h}, \mathrm{H}, \mathrm{A}+\chi_{1}, \mathrm{~h}, \mathrm{H}, \mathrm{A} \rightarrow \mathrm{b} \overline{\mathrm{b}}$ signal (light) and the SUSY (dark) and SM (gray) background with $\mathrm{m}_{\mathrm{A}}=150 \mathrm{GeV} / c^{2}, \tan \beta=5, \mathrm{M}_{2}=350 \mathrm{GeV} / c^{2}, \mu=1000$ $\mathrm{GeV} / c^{2}, \mathrm{~m}_{\tilde{\mathrm{g}}}=1200 \mathrm{GeV} / c^{2}, \mathrm{~m}_{\tilde{\mathrm{q}}}=800 \mathrm{GeV} / c^{2}$ and $\mathrm{m}_{\tilde{\ell}}=500 \mathrm{GeV} / c^{2}$ for $100 \mathrm{fb}^{-1}$. For these parameter values, the Higgs bosons are produced mainly through the $\chi_{2} \rightarrow \mathrm{h}, \mathrm{H}, \mathrm{A}+\chi_{1}$ and $\chi_{1}^{ \pm} \rightarrow \mathrm{H}^{ \pm} \chi_{1}$ decays. The residual $t \bar{t}$ background is also shown in the figure. All neutral Higgs boson signals clearly emerge over the background in this part of parameter space. For larger values of $\mathrm{M}_{2}$ the rate decreases due to the suppression of the $\tilde{\mathrm{q}} \rightarrow \chi_{2} \mathrm{q}, \chi_{1}^{ \pm} \mathrm{q}^{\prime}$ transitions and the damping of the production cross section with increasing gluino mass. The discovery potential for the $\mathrm{A}$ and $\mathrm{H}$ bosons covers the region $\mathrm{m}_{\mathrm{A}} \lesssim 200 \mathrm{GeV} / c^{2}$ and is independent of $\tan \beta$, which makes these channels particularly interesting to cover the range $5 \lesssim \tan \beta \lesssim 10$.

The observability of the lighter scalar Higgs boson $h$ in the SUSY cascades was studied in the mSUGRA framework in [82]. Figure 27 shows the discovery potential for the $\chi_{2} \rightarrow \mathrm{h} \chi_{1}, \mathrm{~h} \rightarrow \mathrm{b} \overline{\mathrm{b}}$ channel in the squark and gluino cascades in the $m_{0}, m_{1 / 2}$ parameter plane with $\mathrm{A}_{0}=0$, $\tan \beta=30$ and $\mu>0$ for 10 and $100 \mathrm{fb}^{-1}$. The regions excluded from theory and by LEP searches [83], isomass curves for the $\mathrm{h}$ and the A Higgs boson and the dominant decay modes are also shown in the figure. The $5 \sigma$-discovery potential, shown in Fig. 27 for the mSUGRA, is valid also in the general MSSM if the mass spectrum is such that the $\tilde{\mathrm{g}} / \tilde{\mathrm{q}} \rightarrow \chi_{2} \rightarrow \chi_{1} \mathrm{~h}$ (or more generally $\tilde{\mathrm{g}} / \tilde{\mathrm{q}} \rightarrow \chi_{i} \rightarrow$ $\left.\chi_{j} \mathrm{~h}\right)$ decays are allowed and the $\chi_{\mathrm{i}} \chi_{\mathrm{j}} \mathrm{h}$ couplings are large enough.

\section{Conclusions}

The present understanding of the CMS potential for the SM and MSSM Higgs bosons have been presented. Some modifications can be expected as full simulation and reconstruction studies will continue in the coming years. The $\mathrm{k}$ factors for both signal and all relevant backgrounds will also be systematically investigated.

For the SM Higgs boson a discovery is possible in the $\mathrm{H} \rightarrow \mathrm{WW}^{*} / \mathrm{WW}$ and $\mathrm{H} \rightarrow \mathrm{ZZ}^{*} / \mathrm{ZZ}$ decay channels in the mass range $130 \mathrm{GeV} / c^{2} \lesssim m_{\mathrm{H}} \lesssim 500 \mathrm{GeV} / c^{2}$ already with an integrated luminosity of $10 \mathrm{fb}^{-1}$ or less and in the mass range $120 \mathrm{GeV} / c^{2} \lesssim m_{\mathrm{H}} \lesssim 1 \mathrm{TeV} / c^{2}$ with an integrated luminosity of $30 \mathrm{fb}^{-1}$. For $\mathrm{m}_{\mathrm{H}} \lesssim 130 \mathrm{GeV} / \mathrm{c}^{2}$, the highest sensitivity (more than $5 \sigma$ for $30 \mathrm{fb}^{-1}$ ) is obtained with the $\mathrm{H} \rightarrow \gamma \gamma$ decay channel in the inclusive and in the $\mathrm{H}+$ jet production, while for $\mathrm{m}_{\mathrm{H}}>130 \mathrm{GeV} / c^{2}$ the $\mathrm{H} \rightarrow \mathrm{WW}^{*} / \mathrm{WW}$ and $\mathrm{H} \rightarrow \mathrm{ZZ}^{*} / \mathrm{ZZ}$ decay channels dominate. The $\mathrm{H} \rightarrow \gamma \gamma$ and $\mathrm{H} \rightarrow \tau^{+} \tau^{-}$decay channels are accessible in the gauge boson fusion production channels with $\mathrm{m}_{\mathrm{H}} \lesssim 150 \mathrm{GeV} / c^{2}$ for $60 \mathrm{fb}^{-1}$. For a $5 \sigma$ statistical significance in the $\mathrm{H} \rightarrow \gamma \gamma$ decay channel in the $\mathrm{WH}$ associated production even higher integrated luminosities are needed. The $\mathrm{H} \rightarrow \mathrm{b} \overline{\mathrm{b}}$ channels yields its highest sensitivity around $\mathrm{m}_{\mathrm{H}} \sim 100 \mathrm{GeV} / c^{2}$. When all channels are combined, a $5 \sigma$ statistical significance can be obtained for $\mathrm{m}_{\mathrm{H}} \geq 114 \mathrm{GeV} / c^{2}$ with an integrated luminosity of $10 \mathrm{fb}^{-1}$.

The lighter scalar MSSM Higgs boson is accessible with $100 \mathrm{fb}^{-1}$ through the inclusive $\mathrm{h} \rightarrow \gamma \gamma$ $\left(\mathrm{m}_{\mathrm{A}} \gtrsim 200 \mathrm{GeV} / c^{2}\right)$ and $\mathrm{h} \rightarrow \mathrm{b} \overline{\mathrm{b}}\left(\mathrm{m}_{\mathrm{A}} \gtrsim 130 \mathrm{GeV} / c^{2}\right)$ decay channels. The $\mathrm{h} \rightarrow \tau^{+} \tau^{-}$channel in the gauge boson fusion process leads to $5 \sigma$ significance for $\tan \beta>4$ and $\mathrm{m}_{\mathrm{A}} \gtrsim 150 \mathrm{GeV} / c^{2}$ already with an integrated luminosity of $30 \mathrm{fb}^{-1}$. The heavy neutral MSSM Higgs bosons are accessible through the $\mathrm{H}, \mathrm{A} \rightarrow \mu^{+} \mu^{-}$and $\mathrm{H}, \mathrm{A} \rightarrow$ $\tau^{+} \tau^{-}$decays channels at large $\tan \beta$. The two-lepton and lepton-plus- $\tau$-jet final states from the $\mathrm{H}, \mathrm{A} \rightarrow \tau^{+} \tau^{-}$decays cover the domain $\mathrm{m}_{\mathrm{A}} \lesssim 300 \mathrm{GeV} / c^{2}$ and $\tan \beta \gtrsim 10$ already with $30 \mathrm{fb}^{-1}$. The two- $\tau$-jet final states extend the sensitivity up to $\mathrm{m}_{\mathrm{A}} \sim 800 \mathrm{GeV} / c^{2}$ with $\tan \beta \gtrsim 35$ for $60 \mathrm{fb}^{-1}$. The heavy scalar $\mathrm{H}$ is accessible also in the $\mathrm{H} \rightarrow \tau^{+} \tau^{-}$channel in the gauge boson fusion for $\mathrm{m}_{\mathrm{A}} \lesssim 120 \mathrm{GeV} / \mathrm{c}^{2}$ with $30 \mathrm{fb}^{-1}$. The heavy charged Higgs bosons can be searched for at large $\tan \beta(\gtrsim 20)$ with the $\mathrm{H}^{ \pm} \rightarrow \tau \nu_{\tau}$ decay channel in the associated production and in the direct production, and with the $\mathrm{H}^{ \pm} \rightarrow$ tb decay channel in the associated production. The $\mathrm{H}^{ \pm} \rightarrow \tau \nu_{\tau}$ decay channel in the associated production yields the highest sensitivity. The light charged Higgs bosons are accessible for $\mathrm{m}_{\mathrm{H}^{ \pm}} \lesssim 140 \mathrm{GeV} / c^{2}$ through $\mathrm{t} \overline{\mathrm{t}}$ production. The intermediate and small $\tan \beta$ range is accessible with the Higgs boson decays to neutralinos in the $\mathrm{H}, \mathrm{A} \rightarrow \chi_{\mathrm{i}} \chi_{\mathrm{j}}$ decay channel with $200 \mathrm{GeV} / c^{2} \lesssim m_{\mathrm{A}} \lesssim 450 \mathrm{GeV} / c^{2}$, $\mathrm{M}_{2} \leq 120 \mathrm{GeV} / \mathrm{c}^{2}$ for $100 \mathrm{fb}^{-1}$. For the Higgs boson production from the gaugino decays in the $\tilde{g}, \tilde{q}$ cascades the region $\mathrm{m}_{\mathrm{A}} \lesssim 200 \mathrm{GeV} / c^{2}$ is accessible already with $30 \mathrm{fb}^{-1}$, independent of $\tan \beta$. For the $\tan \beta$ measurement from event rates in the $\mathrm{gg} \rightarrow \mathrm{b} \overline{\mathrm{b}} \mathrm{H} / \mathrm{A}, \mathrm{H}, \mathrm{A} \rightarrow \tau^{+} \tau^{-}$channels, a precision better than $11 \%$ is expected from the combination the three final states with $\tan \beta \geq 20$ and $\mathrm{m}_{\mathrm{A}}=200 \mathrm{GeV} / c^{2}$ with $30 \mathrm{fb}^{-1}$. For the charged Higgs bosons in the $\mathrm{gb} \rightarrow \mathrm{tH}^{ \pm}, \mathrm{H}^{ \pm} \rightarrow \tau \nu_{\tau}$ channel a precision better than $20 \%$ is expected within the $5 \sigma$-discovery domain with $30 \mathrm{fb}^{-1}$.

Acknowledgements. The authors would like to thank A. Djouadi, S. Moretti, T. Plehn, D. Rainwater, D.P. Roy, M. Spira and D. Zeppenfeld for helpful discussions. One of us (V.D.) would like to thank the European Community for financial support (fellowship HPRN-CT-2002-00326). The authors are grateful to all members of the CMS PRS Editorial Board, P. Janot, I. Van Vulpen, N. Marinelli, N. Neumeister, D. Denegri and V. Hagopian.

\section{References}

1. M. Carena, S. Heynemeyer, C.E.M. Wagner, G. Weiglein, Suggestions for improved benchmark scenarios for Higgsboson searches at LEP2, CERN-TH/99-374, DESY 99186, hep-ph/9912223 
2. M. Carena et al., Nucl. Phys. B 461, 407 (1996)

3. LEP Higgs Working Group, Phys. Lett. B 569, 61 (2003)

4. LEP Higgs Working Group, Searches for the Neutral Higgs Bosons of the MSSM, hep-ex/0107030

5. The LEP Electroweak Working Group, http://lepewwg.web.cern.ch/LEPEWWG/

6. M. Spira, HIGLU, hep-ph/9510347; M. Spira, Fortsch. Phys. 46, 203 (1998)

7. T. Plehn, Charged Higgs Boson Production in BottomGluon Fusion, hep-ph/0206121; E. Boos, T. Plehn, Higgs Boson Production Induced by Bottom Quarks, hepph/0304034

8. S. Slabospitsky, Study of s-Channel Charged Higgs Production in CMS, CMS NOTE 2002/010, hep-ph/0203094

9. M. Carena, J.S. Conway, H.E. Haber, J. Hobbs, Report of the Higgs Working Group of the Tevatron Run 2 SUSY/Higgs Workshop, hep-ph/0010338 and references therein; S. Moretti, J. Rathsman, Pair production of charged Higgs bosons in association with bottom quark pairs at the Large Hadron Collider, hep-ph/0308215

10. R. Rainwater, M. Spira, D. Zeppenfeld, Higgs Boson Production at Hadron Colliders, hep-ph/0203187

11. V. Ravindran, J. Smith, W.L. van Neerven, NNLO Corrections to the Higgs Production Cross Section, hepph/0405302 and references therein

12. A. Djouadi, J. Kalinowski, M. Spira, HDECAY: a program for Higgs Boson Decays in the Standard Model and its Supersymmetric Extension, hep-ph/9704448

13. D. Zeppenfeld, Int. J. Mod. Phys. A 16, 831 (2001); T. Plehn, D. Rainwater, D. Zeppenfeld, Phys. Rev. Lett. 88, 051801 (2002); Phys. Lett. B 454, 297 (1999); M.L. Mangano et al., CERN-TH/2002-287

14. D.P. Roy, Phys. Lett. B 459, 607 (1999)

15. R. Kinnunen, A. Nikitenko, Study of $\mathrm{H} \rightarrow \tau^{+} \tau^{-}$with hadronic $\tau$ decays in CMS, CMS NOTE-2003/006

16. A. Djouadi, Phys. Lett. B 435, 101 (1998)

17. R. Kinnunen, S. Lehti, A. Nikitenko, S. Rantala, Effects of large mixing and light stop for $\mathrm{h} \rightarrow \gamma \gamma$ in MSSM, CMS NOTE 2000/043

18. CMS Collaboration, The Magnet Project, Technical Design Report, CERN/LHCC 97-10, CMS TDR 1, 1997

19. CMS Collaboration, The Tracker Project, Technical Design Report, CERN/LHCC 98-6, CMS TDR 5, 1998

20. CMS Collaboration, CERN/LHCC 2000-016, CMS TDR 5 Addendum 1, 21 February 2000

21. CMS Collaboration, The Electromagnetic Calorimeter Project, Technical Design Report, CERN/LHCC 97-33, CMS TDR 4, 1997

22. CMS Collaboration, The Hadron Calorimeter Project, Technical Design Report, CERN/LHCC 97-31, CMS TDR 2, 1997

23. CMS Collaboration, The Muon Project, Technical Design Report, CERN/LHCC 97-32, CMS TDR 3, 1997

24. CMS Collaboration, CMS Simulation and Reconstruction Package, http://cmsdoc.cern.ch/cmsim/cmsim.html

25. S. Abdullin, A. Khanov and N. Stepanov, CMSJET, CMS TN/94-180

26. V. Drollinger, V. Karimaki, S. Lehti and N. Stepanov, private communication

27. CMS Collaboration, Trigger and Data Acquisition Systems, Technical Design Report, CERN/LHCC 2000-38, CMS TDR 6.1, 2000
28. CMS Collaboration, The Data Acquisition and High-Level Trigger Project, Technical Design Report, CERN/LHCC 2002-26, CMS TDR 6.2, 2002

29. K. Lassila-Perini, Discovery potential of the Standard Model Higgs in CMS at the LHC, Dissertation, ETH No. 12961 (1998)

30. E. Meschi et al., Electron Reconstruction in the CMS Electromagnetic Calorimeter, CMS NOTE 2001/034

31. A. Nikitenko, P. Verrecchia, D. Bomestar, I. Z $\rightarrow \mathrm{e}^{+} \mathrm{e}^{-}$ and $\mathrm{H} \rightarrow 4 \mathrm{e}^{ \pm}$Mass Reconstruction in $\mathrm{PbWO}_{4}$ Crystal ECAL with GEANT simulation of CMS detector; II. Electrons of $\mathrm{E}_{\mathrm{T}}=30 \mathrm{GeV}$ at $\eta=0.1$ and 1.3 in CMS Detector : E/p Matching, Calibration, Resolution, CMS TN/95-019

32. I. Puljak, Potentiel de decouverte du boson de Higgs dans le canal $\mathrm{H} \rightarrow \mathrm{ZZ}^{*} \rightarrow 4 \mathrm{e}^{ \pm}$avec le detecteur CMS. Contribution a la construction du calorimetre electromagnetique de CMS, Dissertation, Ecole Polytechnique, Palaiseau, France, 2000

33. W. Adam et al., Reconstruction of electrons with the Gaussian-sum filter in the CMS tracker at LHC, CMS CR 2003/012

34. I. Iashvili, R. Kinnunen, A. Nikitenko, D. Denegri, Study of the $\mathrm{H} \rightarrow \mathrm{ZZ}^{*} \rightarrow \ell^{+} \ell^{-} \ell^{\prime+} \ell^{\prime-}$ Channel in CMS, CMS TN/95-059 (1995); M. Dzelalija, Z. Antunovic, R. Kinnunen, Study of heavy $\mathrm{H} \rightarrow \mathrm{ZZ} \rightarrow \ell^{+} \ell^{-} \ell^{\prime+} \ell^{\prime-}$ in CMS, CMS TN/95-076

35. C. Charlot, A. Nikitenko, I. Puljak, I. Soric, Comparison of fixed window and clusterization algorithms for $\mathrm{Z} \rightarrow$ $\mathrm{e}^{+} \mathrm{e}^{-}$and $\mathrm{H} \rightarrow 4 \mathrm{e}^{ \pm}$mass reconstruction in $\mathrm{CMS} \mathrm{PbWO}_{4}$ crystal ECAL for Higgs mass 170 and $130 \mathrm{GeV}$, CMS TN/95-101

36. D. Bomestar, D. Denegri, R. Kinnunen, A. Nikitenko, Study of $\mathrm{H} \rightarrow \mathrm{ZZ}^{*} / \mathrm{ZZ} \rightarrow 4 \ell$ with a full GEANT simulation of CMS, CMS TN/95-018

37. J.E. Huth et al., in Proceeding of Research Directions For The Decade: Snowmass 1990, July 1990, edited by E.L. Berger (World Scientific, Singapore, 1992), p. 134

38. D. Green et al., Energy Flow Objects and Usage of Tracks for Energy Measurement in CMS, CMS NOTE 2002/036

39. O. Kodolova, I. Vardanyan, S. Kunori and A. Nikitenko, private communication

40. A. Nikitenko, S. Kunori, R. Kinnunen, Missing Transverse Energy Measurement with Jet Energy Corrections, CMS NOTE 2001/040

41. A. Nikitenko, J. Varela, A study of the 1st Level $\tau$ trigger, CMS TN/95-195

42. S. Eno, A. Nikitenko, R. Kinnunen, private communication

43. R. Kinnunen, A. Nikitenko, Study of $\tau$ jet identification in CMS, CMS NOTE 1997/002

44. S. Eno et al., A Study of a First and Second Level Tau Trigger, CMS NOTE 2000/055

45. D. Kotlinski, A. Nikitenko and R. Kinnunen, Study of a Level-3 Tau Trigger with the Pixel Detector, CMS NOTE 2001/017

46. G. Bagliesi, S. Gennai, G. Sguazzoni, A L2 Trigger for Tau Hadronic Decays with Tracker Isolation in the Low Luminosity Scenario, CMS NOTE 2002/018

47. R. Kinnunen, A. Nikitenko, Study of $\mathrm{H}_{\mathrm{SUSY}} \rightarrow \tau \tau \rightarrow$ $\ell^{ \pm}+\tau$ jet $+\mathrm{E}_{\mathrm{T}}^{\mathrm{miss}}$ in CMS, CMS NOTE 1997/106

48. S. Lehti, Prospects for the Detection of the MSSM Higgs Bosons Decaying into Tau Leptons in the CMS Detector, Dissertation, University of Helsinki, Report Series in Physics, HU-P-D93, 2001 
49. G. Segneri, F. Palla, Lifetime based b tagging with CMS, CMS NOTE 2002 / 046

50. T. Sjostrand et al., High-Energy Physics Event Generation with PYTHIA 6.1, Comput. Phys. Commun. 135, 238 (2001)

51. G. Corcella et al., HERWIG 6: An event generator for hadron emission reactions with interfering gluons (including SUSY processes), JHEP 0101 (2001) 010

52. H. Baer, F.E. Paige, S.D. Protopopescu and X. Tata, hep$\mathrm{ph} / 0001086$

53. A. Pukhov et al., CompHEP - a package for evaluation of Feynman diagrams and integration over multi-particle phase space, INP-MSU 98-41/542

54. A.S. Belyaev et al., CompHEP-PYTHIA interface, Proc. of VII Int. Workshop on advanced computing and analysis techniques in physics research (ACAT2000), Batavia, 2000, hep-ph/0101232

55. K. Hagiwara, D. Zeppenfeld, Nucl.Phys. B 313, 560 (1989)

56. S. Jadach et al., Comput. Phys. Commun. 76, 361 (1993)

57. M. Dittmar, H. Dreiner, LHC Higgs Search with $\ell \nu \ell \bar{\nu}$ Final States, CMS NOTE-1997/083

58. M. Spira, M. Dittmar, Standard Model Higgs cross sections (NLO) and PYTHIA, CMS NOTE 1997/080

59. D. Green et al., Search for the Standard Model Higgs Boson with $\mathrm{M}_{\mathrm{H}} \simeq 170 \mathrm{GeV} / c^{2}$ in $\mathrm{W}^{+} \mathrm{W}^{-}$Decay Mode, CMS NOTE-1998/089, J. Phys. G. Nucl. Part. Phys. 26, 1751 (2000)

60. M.N. Dubinin, V.A. Ilyin, V.I. Savrin, Light Higgs Boson Signal at LHC in the Reactions pp $\rightarrow \gamma \gamma+$ jet and pp $\rightarrow$ $\gamma \gamma+$ lepton, CMS NOTE 1997/101; S. Abdullin et al., Phys. Lett., B 431, 410 (1998)

61. D. de Florian, Z. Kunszt, Phys.Lett. B 460, 184 (1999); C. Balazs, P. Nadolsky, C. Schmidt, C.P. Yuan, Phys.Lett. B 489, 157 (2000)

62. V. Drollinger, Th. Muller, D. Denegri, Searching for Higgs Bosons in Association with Top Quark Pairs in the $\mathrm{H} \rightarrow$ b $\bar{b}$ Decay Mode, CMS NOTE 2001/054, hep-ph/0111312; V. Drollinger, Th. Muller, D. Denegri, Prospects for Higgs Bosons Searches in the Channel $\mathrm{W}^{ \pm} \mathrm{H} \rightarrow \ell^{ \pm} \nu \mathrm{b} \overline{\mathrm{b}}$, CMS NOTE 2002/006, hep-ph/0201249

63. D. Green et al., A Study of $t \bar{t}+\mathrm{H}$ at CMS, CMS NOTE2001/039

64. D. Benedetti, L. Fano, Study of High Level b-trigger Selection of ttH Fully Hadronic Decays, CMS NOTE-2002/044

65. M. Dubinin, Higgs Boson Signal in the Reaction pp $\rightarrow$ $\gamma \gamma+$ two Forward Jets, CMS NOTE 2001/022

66. N. Akchurin et al., Study of Low Mass Higgs Using pp $\rightarrow$ q $\bar{q} \mathrm{H}$ at CMS, CMS NOTE-2002/016
67. G. Azuelos et al., Search for the SM Higgs boson using vector boson fusion at the LHC, Workshop on Physics at TeV Colliders, Les Houches, France, 2001, hep-ph/0203056

68. S. Asai et al., Prospects for the Search for a Standard Model Higgs Boson in ATLAS using Vector Boson Fusion, Eur. Phys. J. C 32, 209 (2003)

69. H.D. Yildiz, Observing Higgs in Weak Boson Fusion with Forward Jet Tagging at the CERN CMS Experiment, Diss. Middle East Technical University, Ankara, Turkey, 2002

70. H.D. Yildiz, M. Zeyrek, R. Kinnunen, Study of $p p \rightarrow j j H$ with $\mathrm{H} \rightarrow \mathrm{WW} \rightarrow \ell \nu \mathrm{q} \overline{\mathrm{q}}$ in CMS for $\mathrm{m}_{\mathrm{H}}=300$ and 600 $\mathrm{GeV} / c^{2}$, CMS NOTE-2001/050

71. L. Bellucci, MSSM Neutral Higgs Boson searches at CMS in the $\mu^{+} \mu^{-}$channel, Dissertation, Universita degli studi di Firenze, December, 2001

72. E. Boos, A. Djouadi, A. Nikitenko, Detection of the neutral MSSM Higgs bosons in the intense-coupling regime at the LHC, CERN TH/2003-152

73. S. Banerjee, M. Maity, Search for the Charged Higgs in Top Decays in CMS, CMS NOTE 2000/039

74. R. Kinnunen, Study of Heavy Charged Higgs in $\mathrm{pp} \rightarrow \mathrm{tH}^{ \pm}$ with $\mathrm{H}^{ \pm} \rightarrow \tau \nu_{\tau}$ in CMS, CMS NOTE 2000/045

75. P. Salmi, R. Kinnunen, N. Stepanov, Prospects of Detecting Massive Charged Higgs from Hadronic Decay $\mathrm{H}^{ \pm} \rightarrow$ tb in CMS, CMS NOTE-2002/024

76. S. Abdullin, D. Denegri, F. Moortgat, Observability of MSSM Higgs bosons via sparticle decay modes in CMS, CMS NOTE 2001/042

77. M. Bisset, F. Moortgat, S. Moretti, Trilepton+top signal from chargino-neutralino decays of MSSM charged Higgs bosons at the LHC, CMS NOTE 2003/004, hepph/0303093

78. B. Di Girolamo et al., Experimental Observation of an invisible Higgs Boson at LHC, Summary Report (2001), Workshop on Physics at TeV Colliders, Les Houches, France, 2001, hep-ph/0203056

79. O.J.P. Eboli, D. Zeppenfeld, Phys. Lett. B 495, 147 (2000)

80. G. Belanger et al., Phys. Lett. B 519, 93 (2001)

81. A. Datta, A. Djouadi, M. Guchait, F. Moortgat, Detection of MSSM Higgs bosons from SUSY particle cascade at the LHC, hep-ph/0303095

82. S. Abdullin, D. Denegri, On the Possibility to Observe $\mathrm{h} \rightarrow \mathrm{b} \overline{\mathrm{b}}$ with $\mathrm{S} / \mathrm{B} \sim 1$ in SUSY (mSUGRA), and Implications for Tracker and HCAL CMS NOTE-1997/070

83. OPAL Collaboration, Search for nearly mass degenerate Charginos and neutralinos at LEP, CERN-EP-2002-063, Submitted to Eur. Phys. J; ALEPH Collaboration, Search for charginos nearly mass degenerate with the lightest neutralino in $\mathrm{e}^{+} \mathrm{e}^{-}$collisions at centre-of-mass energies up to $209 \mathrm{GeV}$, Phys.Lett. B 533 (2002) 